\title{
Steady and Unsteady Excitation of Separated Flow over the NASA Hump Model
}

\author{
Mehti Koklu ${ }^{1}$ \\ NASA Langley Research Center, Hampton, VA 23681
}

\begin{abstract}
Separated flow and its control over the NASA hump model were investigated at subsonic speeds. Three-dimensional, unsteady fluid dynamic simulations were supplemented by wind tunnel measurements. Flow control was implemented by means of spatially distributed discrete jets operating in steady and unsteady modes. The effects of excitation amplitude and frequency were studied numerically and experimentally. Several integral parameters were explored as quality metrics. In addition to the existing ones, two new integral parameters, which are slightly modified versions of the normal force and moment coefficients, were introduced. These two parameters together with pressure drag coefficient were found to be well correlated with the flow control and used in the performance evaluation of different flow control methods including, zero net mass flux actuators, steady suction, sweeping jet actuators, and the currently studied steady and unsteady excitations. For the cases tested, it was found that the unsteady excitation is superior to the steady excitation and slightly better than the sweeping jet actuators whereas the steady suction was found to be the most effective. Although the numerical simulations overpredict the separation bubble, these simulations capture the salient features of flow separation control and hence help us to understand the effect of steady/unsteady excitation on the separated flow.
\end{abstract}

\section{Nomenclature}

$\begin{array}{ll}c & =\text { hump chord } \\ C_{d p} & =\text { pressure drag coefficient } \\ C_{i n v} & =\text { inviscid flow comparison coefficient } \\ C_{m q} & =\text { moment coefficient based on quarter-chord } \\ C_{m x} & =\text { moment coefficient based on } x_{m} \\ C_{\mu} & =\text { jet momentum coefficient } \\ C_{n} & =\text { normal force coefficient } \\ C_{n p} & =\text { modified normal force coefficient } \\ C_{p} & =\text { pressure coefficient } \\ \operatorname{Re}_{c} & =\text { Reynolds number based on hump chord } \\ x, y, z & =\text { streamwise, vertical, and spanwise directions } \\ x_{m} & =\text { hump slope discontinuity } \\ x_{r} & =\text { reattachment location } \\ x_{s} & =\text { separation location }\end{array}$

\section{Introduction}

Understanding flow separation and its control via an actuator has been the subject of much research from the beginning of the twentieth century. In parallel to many experimental studies, the last century witnessed the exponential growth of numerical simulations of separated flows due to the availability of computational resources. Accurate modeling and prediction of fluid flows around bodies could change the design and hence the performance of a fluid machinery system. Among many wind tunnel models that can be simulated with reasonable computational resources, the wallmounted hump model is a good candidate to investigate flow separation and its control. This model was one of the case studies in the Computational Fluid Dynamic (CFD) Validation of Synthetic Jets and Turbulent Separation Control Workshop in 2004 (CFDVAL2004) and is a well-known benchmarking case for CFD validations. It is well

\footnotetext{
${ }^{1}$ Aerospace Engineer, Flow Physics and Control Branch, MS 170, Member AIAA.
} 
documented experimentally ${ }^{1-6}$ and numerically (see Workshop summary paper by Rumsey et al. ${ }^{7}$ ) over a wide range of Reynolds numbers $\left(0.4 \times 10^{6}<R e_{c}<26 \times 10^{6}\right)$. One of the key advantages of this model is that the separated flow over the hump model is sensitive to flow control, ${ }^{2-4,6}$ which allows the study and comparison of different flow control concepts, both numerically and experimentally. Although the accurate prediction of the massively separated flows is still challenging, CFD simulations provide key contributions in understanding mechanisms associated with flow separation and its control when using different flow control techniques.

The wall-mounted hump model is the suction side of the GLAS (Glauert Laminar Airfoil Section) II airfoil. The original GLAS II airfoil was designed to provide large lift coefficients (up to 2) without having a flap. The airfoil's large thickness ( $31.5 \%$ of chord) enabled a favorable pressure gradient and hence laminar flow over the most of the airfoil surface. ${ }^{1}$ A large lift coefficient was achieved using boundary layer control from a two-dimensional slot located at $69 \%$ of the chord. Both steady suction and steady blowing were tested at low $\left(0.94 \times 10^{6}\right)$ and high $\left(2.9 \times 10^{6}\right)$ Reynolds numbers and the steady blowing appeared to be less effective than suction, requiring a larger mass flow to reattach the flow over the ramp section. ${ }^{2}$ The upper surface of the GLAS II airfoil was also used by Goldschmied et al. ${ }^{8}$ in a body of revolution to generate self-propelled (i.e., static pressure thrust) body with the use of suction boundary layer control from the two-dimensional axisymmetric slot.

The wall-mounted hump model was initially tested at high Reynolds numbers $\left(2.4 \times 10^{6}-26 \times 10^{6}\right)$ in a cryogenic wind tunnel facility. ${ }^{6}$ One of the outcomes of the cryogenic wind tunnel test is that the separated flow and its control are negligibly sensitive to Reynolds number and the incoming boundary layer. This finding is important because it allows the wind tunnel results to be scaled. The steady suction, steady blowing, and periodic excitation (all from the two-dimensional slot) were used to control flow separation at high Reynolds numbers. It was shown that the periodic excitation is comparable to the steady suction and significantly more effective than the steady blowing. In addition, two different slot locations were tested and the slot location close to flow separation ( $64 \%$ of chord) was found to be more effective than the upstream location (59\% of chord). ${ }^{6}$

Negligible sensitivity to Reynolds number motivated the testing of the wall-mounted hump model in a low speed wind tunnel to provide more detailed measurements as part of the CFD Validation Workshop. In these low Reynolds number $\left(0.94 \times 10^{6}\right)$ tests, the original hump model was modified to have a reduced thickness $(\approx 13 \%$ of the chord $)$. The detailed measurements of the baseline configuration and flow separation control using the steady suction and the oscillatory blowing with zero net mass flux (ZNMF) actuators from the two-dimensional slot were reported in Refs. 2 and 3, respectively. Although the main motivation of the study was not to eliminate the separation bubble, the baseline and flow control cases will be used herein for comparisons.

The wall mounted hump model has been frequently used in the numerical studies, mostly, to validate different numerical algorithms. ${ }^{7}$, 9-12 The hump model has also gained recent attention in the active flow control (AFC) community with an emphasis to test different flow control concepts. For example, passive separation control with vortex generators (VG) and active separation control with steady discrete blowing were studied both experimentally and numerically and the results showed that the VGs substantially reduced, but could not eliminate, flow separation. ${ }^{13-}$ ${ }^{14}$ In another experimental study, the sweeping jet (SWJ) actuators were applied on the hump model and the results were compared to the Workshop data. It was found that the SWJ actuators provide better control authority than the tested flow control methods (suction \& ZNMF actuators) applied in the Workshop, especially for high amplitude excitations. ${ }^{14}$ The superiority of the SWJ actuators over discrete round jets was reported in another experimental study. ${ }^{15}$ A different SWJ actuator configuration was numerically evaluated by Kara et al., ${ }^{16}$ where the sweeping jet actuators were found to reduce the flow separation as the actuator mass flow increases.

The present study is aimed at improving our understanding of separated flows and its control via steady/unsteady excitation. The steady/unsteady excitation from discrete nozzles is used to control flow separation over the wall mounted hump model. Three-dimensional, unsteady simulations are carried out in parallel with the wind tunnel experiments. Although this model has been used as a turbulence model validation case by CFD researchers, it should be noted that this study is not a code development/validation study, but a combined computational/experimental effort to better understand the separated flow over the hump model and its control with steady/unsteady excitation. Several integral parameters are explored to evaluate the flow control configurations. In addition, the performance assessment of different flow control methods including the current and previously tested methods on the hump model (ZNMF actuators, ${ }^{4}$ steady suction, ${ }^{3,6}$ steady blowing, ${ }^{6,13}$ unsteady blowing, ${ }^{6}$ and SWJ actuators ${ }^{14,15}$ ) is performed using these integral parameters.

\section{Description and Procedure}

This section contains a brief description of the experimental and numerical setups and various integral parameters as quality metrics to evaluate the performance of the flow control methods applied to the wall-mounted hump model. 


\section{A. Experimental Setup}

The wall-mounted hump model used in this study is the same model that was used by Greenblatt et al. ${ }^{2,4}$ during the CFDVAL2004 Workshop in 2004. The experimental configuration was also similar to that of the Workshop and the detailed description of the model and the wind tunnel can be found in Ref. 3. Only a brief summary will be given for completeness. The experiments were conducted in the NASA Langley 20 x 28 in. wind tunnel at a freestream Mach number of 0.1 . The characteristic length was defined as the hump chord (c), which is $420 \mathrm{~mm}$. The Reynolds number based on the hump chord was $R e_{c}=0.94 \times 10^{6}$. The model was mounted on a splitter plate where the boundary layer was tripped near the leading edge using $20 \mathrm{~mm}$ thick (\#60) sand paper. The hump model was installed between two endplates to reduce the effects of tunnel sidewall boundary layers and associated corner vortices on each side. The CAD rendering of the hump model (without endplates) is shown in Fig. 1. The hump model used in the Workshop had a two-dimensional slot located at $x / c=0.65$ that was connected to an interior plenum between the forebody and ramp. As illustrated in this figure, the model midsection, which comprised the two-dimensional slot, was refabricated using high-resolution stereolithography to accommodate an actuator array. The actuator array consisted of a single row of 31 jet nozzles with $16.5 \mathrm{~mm}$ spacing (inset in Fig. 1) spanning the entire model width $(584 \mathrm{~mm})$. Jet-nozzle exits were located at $x / c=0.65$ similar to the previous studies..$^{3-4,9-15}$ The nozzles were arranged in a lateral line pattern with the middle actuator located at the model centerline. The nozzles were connected to a common plenum by individually controlled high-speed valves, which were used to generate steady/unsteady excitations. In the experiments, the nozzle inlets were connected to the high-speed valves via circular tubing. Therefore, the inlet of the nozzles begins with a circular shape that smoothly transitions to first a square and then to a rectangular shape without changing the cross sectional area (see Fig. 1 inset). Transitioning from a square cross section to a rectangular cross section generated a diffuser type nozzle, which resulted in a $( \pm)$ skew angle on each side of the nozzles. Due to the skew angles, the jets were seen to produce weak streamwise vortices. ${ }^{13}$ The nozzle exit dimensions were approximately $1 \mathrm{~mm}$ by $5.6 \mathrm{~mm}$. The jet axis of the nozzle pointed parallel to the freestream flow; however, the angle between the jet axis and local flow was approximately $20^{\circ}$ due to the surface curvature at this location. The flow rate to the actuator array was controlled by an electronic pressure regulator and monitored by a commercial flow meter.

\section{B. Computational Setup}

A Lattice Boltzmann Method $(\mathrm{LBM})^{17}$ based commercial software package was used to simulate the threedimensional unsteady flow over the hump model. A modified $\mathrm{k}-\epsilon$ turbulence model, which involves a very large eddy simulation approach, was used to resolve the large energy containing scales. In addition, appropriate wall functions ${ }^{18}$ were utilized to capture the boundary layer behavior without excessive grid resolution near the solid walls. The LBM equations were solved on embedded Cartesian meshes (voxels), which were successively refined near the high gradient regions (e.g., shear layers) using variable resolutions. The model used an explicit time advancement scheme that allowed massively parallel simulations.

The computational modeling included the three-dimensional hump geometry, endplates, splitter plate, and the jet nozzles. The length of the splitter plate upstream of the model was similar to the experiment (4.6c) in contrast to the previous simulations (see Ref. 7) that lengthened the splitter plate (to 6.4c) in order to match the boundary layer profile at the inflow location $(x / c=-2.14)$. In the simulations, a viscous no-slip boundary condition was imposed on solid walls (hump geometry, endplates, splitter plate), whereas the tunnel sidewalls and the ceiling were treated as inviscid walls to eliminate the additional cost of resolving boundary layers. Constant velocity (Mach number of 0.1 ) inlet and atmospheric pressure outlet boundary conditions were prescribed at the inflow and outflow, respectively. For the steady excitation cases, a constant mass flow boundary condition was used at the nozzle inlets to generate steady discrete jets. For the unsteady excitation cases, an unsteady (square wave type) mass flow boundary condition was used at the nozzle inlets to generate pulsed type unsteady jets.

A mesh dependence study was performed prior to the numerical simulations to confirm that the solutions were independent of the grid resolution (not reported here). For this purpose, the baseline-separated flow was simulated at four different (coarse, medium, fine, finest) grid resolutions that had 17, 27, 53, 92 million fine equivalent voxels, respectively. The fine and finest grid resolutions provided relatively close results; therefore, it is anticipated that at least a fine grid resolution with 53 million fine equivalent voxels is required to provide a grid independent solution. The smallest voxel size for the fine grid resolution was $0.4 \mathrm{~mm}$. The physical time step $\left(6.87 \times 10^{-7} \mathrm{sec}\right.$. in this case) was automatically adjusted depending on the finest voxel size. The simulations were carried out 10 characteristic times. The state of the simulations was monitored using the variables recorded at the probe locations at very high frequency. Since the solutions were initialized from rest, the data for the first three characteristic times were discarded in order to remove the effects of the initial transient phase. 


\section{Integral Parameters for Quality Metrics}

Performance evaluation of different flow control configurations requires a quality metric. Various quality metrics have been proposed in the literature to compare different flow control actuators that incorporate the lift, drag, actuator energy, etc. ${ }^{19}$ For the three-dimensional experimental models (such as wing or bluff body), it is relatively easy to evaluate these metrics, as the lift and drag values are typically available. However, for the wall-mounted models that create flow separation due to an adverse pressure gradient (such as hump, bump, ramp step, etc.), the model lift and drag values are typically not available. The wind tunnel tests usually provide pressure distribution and flow reattachment location (either from flow visualization or from the fluctuating pressures) together with the local velocity measurements using velocimetry techniques. Therefore, a candidate quality metric should be based on the pressure distribution. Recently, Borgmann et al. ${ }^{15}$ used a metric based on the pressure distribution for the hump model. In this metric, the obtained pressure distribution using flow control was compared to the ideal (i.e., inviscid solution) and baseline cases. This inviscid flow comparison coefficient $\left(C_{i n v}\right)$ was given as follows: ${ }^{15}$

where

$$
C_{i n v}=\frac{\int_{c} \Delta C_{p m} d(x / c)}{\int_{c} \Delta C_{p i} d(x / c)}
$$

$$
\begin{gathered}
\Delta C_{p i}=\left|C_{p, \text { ideal }}-C_{p, \text { baseline }}\right| \\
\Delta C_{p m}=\operatorname{sign}\left(\Delta C_{p i}\right)\left(C_{p}-C_{p, \text { baseline }}\right)
\end{gathered}
$$

$C_{p, \text { ideal }}$ is the inviscid solution, $C_{p, \text { baseline }}$ is the measured baseline pressure distribution, and $C_{p}$ is the analyzed pressure distribution. In addition, the authors incorporated the amount of energy consumed by the actuators. By definition, this metric indicates the efficiency of an actuator relative to the inviscid and baseline cases. Therefore, $C_{i n v}$ is the measure of how close the analyzed pressure distribution is to the ideal case from its baseline. As shown in these equations, this metric requires both numerical and experimental pressure distributions.

The geometry of the wall-mounted hump model given in Fig. 2 helps us to better understand the design features of the model as well as the integral parameters used. As shown in this figure, the hump model was designed to have a large slope in the first quarter of the model $(x / c<0.25)$ that generates a favorable pressure gradient and then a positive but diminishing slope until the model midchord $(x / c=0.5)$ where the slope becomes negative. After the model midchord, the model was designed to have a small but negative slope that generates a mild adverse pressure gradient until $x / c=0.65$, where the actuator exits are located. A large negative slope after this point generates a strong adverse pressure gradient, which gradually reduces and finally levels out at the model trailing edge. The surface curvature plot indicates a discontinuity at $x_{m}=x / c=0.656$, where flow normally separates in the baseline case. The discontinuity at this location is the outcome of Glauert's design to provide the shortest possible pressure recovery by using boundary layer control. This location could be used in the quality metrics as it separates two distinctive flow regions that are expected from successful flow control applications. Upstream of this point $\left(x / c<x_{m}\right)$, the model should provide as high suction pressure as possible, whereas the pressure should be recovered without any flow separation downstream of it.

Below are the commonly used integral parameters to evaluate the effectiveness of any particular flow configuration:

$$
C_{n}=-\int_{c} C_{p} d(x / c), \quad C_{d p}=\int_{c} C_{p} d(y / c), \quad C_{m q}=\int_{c} C_{p}(x / c-0.25) d(x / c)
$$

The first integral parameter is the normal force coefficient that is similar to the lift coefficient of an airfoil at zero deg. angle of attack without having the lower surface pressures. The second integral parameter is the pressure (or form) drag coefficient, which is similar to the drag coefficient without the viscous effects. The pressure drag coefficient depends on separated flow; therefore, it will give a measure of reduction of the separation bubble, and hence the effect of flow control. The third integral parameter is the moment coefficient about the quarter-chord of the model. Usually, the moment coefficient is defined as the moment produced by the aerodynamic forces (here pressure) based on the aerodynamic center, that is typically near $25 \%$ of the chord. On an airfoil, $C_{m q}$ usually corresponds to the pitching moment that should be close to zero for a safe operation. In fact, the aerodynamic center of the original GLAS II airfoil was reported as $31 \%$ of the chord. ${ }^{1-2}$ In addition to these well-known integral parameters, two new integral parameters are introduced in this study:

4

American Institute of Aeronautics and Astronautics 


$$
C_{n p}=-\int_{c} C_{p} \operatorname{sign}\left(x / c-x_{m}\right) d(x / c), \quad C_{m x}=\int_{c} C_{p}\left(x / c-x_{m}\right) d(x / c)
$$

These parameters are motivated by the expected outcome from a flow control over the hump model. A successful flow control should produce higher suction pressure upstream and better pressure recovery downstream. In order to satisfy this requirement, the normal force and moment coefficients in Eq. 1 were slightly modified. The fourth integral parameter, $C_{n p}$, is a slight variation of the normal force coefficient. In an ideal case, the flow over the hump model was designed to produce suction pressure over most of the upper surface and pressure recovery over a relatively shorter distance $\left(x / c>x_{m}\right)$. An increase in the normal force coefficient implies more suction pressure but less pressure recovery. Therefore, the normal force coefficient comprises only the effect of suction pressure but not the pressure recovery downstream. By changing the sign of the integral for $x / c>x_{m}$, this integral parameter also includes the effect of the pressure recovery. Therefore, the modified version of the normal force coefficient, $C_{n p}$, should indicate higher suction pressures over the forward body $\left(x / c<x_{m}\right)$ and better pressure recovery over the aft body $\left(x / c>x_{m}\right)$ as expected from a successful flow control. The fifth integral parameter, $C_{m x}$, is similar to the moment coefficient in Eq. 1 but it is about $x_{m}$ instead of the model quarter chord. Again, a reduction in the quarter-chord moment coefficient implies more pressure recovery downstream but less suction pressure upstream. Therefore, $C_{m q}$ comprises only the effect of pressure recovery but not the suction pressure upstream. By moving the moment center to $x_{m}$, an increase in this parameter not only implies more pressure recovery downstream but also more suction pressure upstream as expected from a successful flow control.

On a typical three-dimensional model, these parameters are usually integrated from the leading edge to the trailing edge where the upper and lower surface pressures meet. In this study, because the model is on the splitter plate, the pressure distributions are integrated from the leading edge of the model to $x / c=1.5$, where the pressure values reach equilibrium with the tunnel pressures.

\section{Results}

The baseline separated flow over the hump model was previously reported numerically and experimentally in Refs. 13 and 14. It was found that the boundary layer measurement at the inflow location $(x / c=-2.14)$ was considerably thinner than the reported boundary layer data in the Workshop (19 $\mathrm{mm}$ and $30.5 \mathrm{~mm}$, respectively). The reason behind this discrepancy in the incoming boundary layer was attributed to a possible separation bubble at the leading edge of the splitter plate due to the blockage created by the suction manifold used in the CFDVAL2004 experiments. ${ }^{14}$ The simulated boundary layer profile was in good agreement with the new boundary layer measurements without extending the splitter plate to $-6.4 c .^{13}$ Although the incoming boundary layers were different in the two experiments, the surface pressure distributions were in very good agreement with the data reported in the Workshop. ${ }^{13-14}$ Numerical simulations could not accurately predict the size of the separation bubble compared to the experiment. The baseline flow reattachment was predicted to be at $x / c=1.266,{ }^{13}$ but was reported at $x / c=1.15$ (using oilflow visualization) in the experiment. ${ }^{14}$ The longer separation bubble resulted in a shift in the pressure and skin friction distributions in the streamwise direction.

The baseline separated flow over the hump model is presented in the simulated surface flow visualization (Fig. 3). Near surface streamlines are superimposed onto the contours of velocity magnitude to show the three-dimensional flow topology over the hump model. Note that the endplates were removed during the post-processing in order not to block the view. The incoming flow over the splitter plate decelerates near the model leading edge. The decelerated flow rapidly accelerates over the first half of the model due to the favorable pressure gradient (shown as higher velocity magnitudes) and then the flow starts to decelerate until the flow separates near $x / c=0.66$. Flow separation is nominally two-dimensional except near endplate regions due to the corner vortices. The separation bubble appears as two symmetric corner vortices that fill the entire model width. Although the corner vortices were reported to be much smaller in the experiment, ${ }^{14}$ the lateral flow along the reattachment line was also highlighted in the surface oilflow visualizations, which indicates the existence of two symmetric corner vortices that extend over the entire separation bubble. The flow reattachment is at $x / c=1.266$ near the centerline and at $x / c=1.12$ downstream of the corner vortices. This is more than a $12 \%$ deviation in the flow reattachment across the model span. The reattachment locations were reported to be at $x / c=1.15$ and $x / c=1.11$, near the centerline and downstream of corner vortices in the experiment, respectively. ${ }^{14}$ 
Figure 4 shows the centerline pressure $\left(C_{p}\right)$ distribution for the baseline case predicted by the numerical simulations over the hump model compared to the experiment. Flow deceleration $(x / c<0)$, flow acceleration $(0<x / c<0.5)$, a suction peak $(x / c \approx 0.5)$, and flow separation at $x / c \approx 0.66$ (indicated by a plateau in the $C_{p}$ distribution) are observed in the pressure distributions. Overall, the simulated $C_{p}$ distribution agrees well with the experiment except in the pressure recovery region. The trend of the pressure recovery is also similar to that of the experiment but due to the larger separation bubble predicted by numerical simulations, the pressure recovery curve is shifted in the streamwise direction. The off-center pressure distribution $(z / c=0.5$, along the line passing the reattachment node) shows the effect of three-dimensional flow separation on the $C_{p}$ distribution. As indicated in the surface flow visualization (Fig. 3 ), the flow reattaches sooner due to the corner vortices. This results in quicker pressure recovery confirming the offset between the numerical and experimental results is due to the longer separation bubble. The inviscid solution of the flow over the three-dimensional hump model is also presented in this figure. The inviscid (i.e., ideal) flow over the hump model results in more flow deceleration near the leading edge of the hump model and more acceleration afterward. There is a consistent increase in the suction pressures reaching up to $C_{p}=-1.5$ (almost $50 \%$ more than that of the separated flow case) at $x / c=0.66$, where the flow normally separates in the baseline case. After the peak, the suction pressure suddenly drops and recovers to the tunnel pressure downstream. Although an ideal case, the inviscid flow solution will be used as a target for the flow control comparisons.

The integral parameters calculated using the pressure distributions (in Fig. 4) are given in Table 1. As mentioned before, the first parameter, $C_{i n v}$, is the measure of how close the obtained pressure distribution to that of the inviscid solution $\left(C_{i n v}=1\right)$ from the baseline case $\left(C_{i n v}=0\right)$. Numerical simulations of the baseline case resulted in negative $C_{i n v}$ values indicating that the pressure distribution predicted by CFD is worse (in terms of closeness to the inviscid solution) than the experiment due to the inaccurate prediction of the separation bubble. The second integral parameter is the pressure drag coefficient. The ideal flow over the hump generated net thrust $\left(C_{d p}=-0.013\right)$. This is consistent with the design purpose of the hump model, which was also used by Goldschmied et al. ${ }^{8}$ to generate thrust by controlling the flow separation. The separated flow substantially increases the pressure drag coefficient (to 0.031 ). CFD predicted a lower pressure drag coefficient than the experiment although the separation bubble is predicted to be longer and $C_{i n v}$ indicates a worse $C_{p}$ distribution. Interestingly, the lower drag in the simulations (note that it is total drag including viscous and pressure drag) was found to be well correlated with the elongation of the separation bubble in the numerical simulations of Yeh et al. ${ }^{20}$ The lower amount of drag may also be due to the pressure distribution in the third quadrant of the model $(0.5<x / c<0.75)$, which contributes most to the drag coefficient, being slightly higher than the experiment. Again in this region $(0.5<x / c<0.75)$, the off-center pressure distribution is lower than the centerline pressure resulting in a higher drag coefficient (0.029) than the centerline although the separation bubble is shorter due to the corner vortices (see Fig. 3).

Table 1. Integral parameters for the baseline case.

\begin{tabular}{lcccc}
\hline \hline & $\begin{array}{c}\text { Inviscid } \\
\text { Flow }\end{array}$ & $\begin{array}{c}\text { Baseline } \\
\text { Exp. }\end{array}$ & $\begin{array}{c}\text { Baseline } \\
\text { CFD }\end{array}$ & $\begin{array}{c}\text { Baseline } \\
\text { Off-center }\end{array}$ \\
\hline$C_{i n v}$ & 1.000 & 0.000 & -0.130 & -0.110 \\
$C_{d p}$ & -0.013 & 0.031 & 0.022 & 0.029 \\
$C_{n}$ & 0.419 & 0.564 & 0.610 & 0.635 \\
$C_{n p}$ & 0.506 & 0.161 & 0.118 & 0.123 \\
$C_{m q}$ & -0.065 & -0.200 & -0.240 & -0.246 \\
$C_{m x}$ & 0.105 & 0.029 & 0.007 & 0.012 \\
\hline \hline
\end{tabular}

The normal force coefficient, $C_{n}$, which is similar to the lift coefficient of an airfoil at zero degree angle of attack, may result in misleading conclusions. As shown in the table, even the inviscid flow produces lower $C_{n}$ (or lift coefficient) values than the baseline separated flow, which is counterintuitive. This is because for a typical airfoil, controlling flow separation generates higher circulation and hence higher lift coefficient by moving the stagnation point to the lower surface and by turning the flow down at the trailing edge. For a wall-mounted model, the stagnation point cannot move and the flow at the trailing edge cannot turn due to the presence of the splitter plate in the front and aft of the model. Therefore, the normal force coefficient is not a good indicator for typical wall-mounted models. A slightly modified version of the normal force coefficient (given in Eq. 2) can be used instead. The normal force coefficient with the pressure recovery effect, $C_{n p}$, not only reflects higher suction pressures upstream but also more 
pressure recovery (lower suction pressures) downstream. As shown in the table, $C_{n p}$ is the highest $(0.506)$ for the inviscid case and the lowest $(0.118)$ for the baseline separated flow predicted by numerical simulations. Since the experimental baseline case produced a shorter separation bubble, the $C_{n p}$ coefficient is slightly larger than that of CFD. The same conclusion applies to the $C_{n p}$ coefficients of the centerline and off-center pressure distributions.

The moment coefficient about the quarter chord, $C_{m q}$, is similar to the pitching moment coefficient for most airfoils as the aerodynamic center is usually close the quarter chord location. Although it has been frequently used in airfoil studies, the $C_{m q}$ coefficient may give misleading conclusions for the wall-mounted models, just like the normal force coefficient. As shown in Fig. 4, the pressures upstream and downstream of the flow separation location have the same sign. Decreasing $C_{m q}$ implies not only increased pressure recovery downstream but also reduced suction pressures in the second quadrant of the model. The moment coefficient has been found to be correlated with the applied flow control in some cases (e.g., the cases in Table 1). This is because the moment arm of the pressure distribution over the separation bubble is longer than that of the suction pressure upstream and hence its effect is more dominant. There might be some cases in which $C_{m q}$ results in misleading conclusions. For example, the $C_{m q}$ values of the simulated centerline and off-center pressure distributions indicate that the centerline pressure distribution is better than that of the off-center location. However, Fig.3 clearly showed that the off-center separation bubble is shorter (controlled by the corner vortex upstream) than that of the centerline. This is not the case for three-dimensional airfoils, as the center of pressure is also close to the quarter chord meaning that the suction pressures are dominantly distributed over the first quarter of the chord. Selecting the point of surface discontinuity, $x_{m}$, as the moment center (i.e., $C_{m x}$ ) implies higher suction upstream and better pressure recovery downstream. Due to the moment arm length, $C_{m x}$ also accounts for the higher suction pressures near the leading edge of the model and lower suction pressure (or positive pressure) downstream, as the latter will be the measure of the total pressure recovery of the system. In addition, it is more forgiving to the local pressure discontinuities near the separation line when flow control is applied, as the moment arm is smaller and hence its effect on the total moment coefficient is lower. As a result, it is not very sensitive to the local variations in the pressure distributions where the flow separates. Similar to the $C_{n p}$ coefficient, the goal of flow control is to increase $C_{m x}$. As shown in the table, inviscid flow provided the highest $C_{m x}$ and the baseline flow predicted by numerical simulations at the centerline provided the lowest value. Since the $C_{d p}, C_{n p}$, and $C_{m x}$ coefficients are better correlated with the state of the flow, they will be used as the quality metrics in the performance assessments. In addition, although the flow over the hump model was shown to be three-dimensional in Fig. 3, only the centerline pressure distributions will be used in this study for the evaluation of the flow control methods, as the experimental results are only available at the centerline.

An uncertainty estimate was performed for the baseline case using the AIAA standards defined in Ref. 21. In the experiments, the nondimensional pressure coefficient $\left(C_{p}\right)$ was obtained by dividing the differential pressure (measured with an electronic pressure scanner system) by the tunnel dynamic pressure (measured with a high accuracy differential pressure sensor). The uncertainty propagation of each measurement system to the nondimensional pressure and the integral coefficients were calculated using the accuracies given by the manufacturers. The precision limits of both measurement systems are about two orders of magnitude less than the bias limit; therefore, they were not included in the uncertainty estimations. The uncertainty of the $C_{p}$ coefficient is not absolute but a function of $C_{p}$, and the maximum uncertainty in $C_{p}$ was found to be as \pm 0.004 . The uncertainty of the $C_{p}$ distribution was then incorporated into the integrals given in Eq.1 and 2 as described in Ref. 21. The uncertainties of the integral parameters were obtained as $\pm 0.0008, \pm 0.0008$, and \pm 0.0002 , for the $C_{n p}, C_{m x}, C_{d p}$, coefficients, respectively.

\section{A. Flow Control with Steady Excitation}

Steady excitation from 31 discrete nozzles was achieved by fully opening the high-speed valves and adjusting the flow rate to the common plenum to a desired level. In this study, the nondimensional momentum coefficient, $C_{\mu}$, is used to characterize the flow control configurations. The momentum coefficient has been used as a scaling parameter in the literature and it is the ratio of the fluid momentum injected by the actuator to the free stream dynamic force:

$$
C_{\mu}=\frac{\dot{m} U_{j e t}}{1 / 2 \rho U_{\infty}^{2} A_{r e f}}
$$

where $\dot{m}$ is the mass flow rate to the actuator array, $U_{j e t}$ is the averaged jet velocity at the exit and $A_{\text {ref }}$ is the projected model area.

An illustration of the three-dimensional turbulent structures with the flow control using steady excitation is presented in Fig. 5. The momentum coefficient for this particular case is $0.24 \%$. In this figure, instantaneous isosurfaces of $\lambda_{2}$ - criterion were colored by the streamwise velocity to highlight the vortex cores. Since this level of 
flow control authority is not sufficient to keep the flow attached over the hump model, ${ }^{3}$ the shear layer over the hump model is separated. The separated shear layer sheds from the hump surface resulting in the formation of a wide variety of three-dimensional turbulent structures. These vortical structures convect and eventually diffuse downstream (not shown).

Application of steady excitation from 31 discrete nozzles initially increases the suction pressure beginning from the flow relaxation region $(x / c \sim 0.2)$ until the suction peak at $x / c=0.61$ as illustrated by the experimental pressure distributions (Fig. 6). The flow continues to accelerate despite the mild adverse pressure gradient between $x / c=0.5$ and $x / c=0.61$. The suction peak at $x / c=0.61$ is followed by a sharp pressure drop. The plateau in the pressure distribution near $x / c=0.70$ possibly indicates the existence of a flow separation bubble. Compared to the baseline case, the steady blowing resulted in substantial increase in the suction pressure as well as in the pressure recovery. By gradually increasing the flow rate, the effect of momentum coefficient was studied. The flow control authority increases proportionally with the momentum coefficient, providing higher suction pressures upstream and better pressure recovery downstream. Interestingly, although increasing the momentum coefficient resulted in a proportional offset in the suction pressures as well as in the pressure recovery, its effect is minimal near flow separation. As shown in this figure, even doubling or tripling the momentum coefficient could not change the local pressure values at this location (near $x / c=0.70$ ). This is possibly due to the severe adverse pressure gradient that fixed the flow separation location for this level of excitation. In addition, since the jet axis is not tangential to the surface, the steady excitation may also amplify the local separation bubble.

Table 2. Variation of integral parameters with steady excitation amplitude.

\begin{tabular}{cccccc}
\hline \hline & Baseline & $C_{\mu}=0.11 \%$ & $C_{\mu}=0.18 \%$ & $C_{\mu}=0.24 \%$ & $C_{\mu}=0.3 \%$ \\
\hline$C_{n p}$ & 0.161 & 0.243 & 0.270 & 0.281 & 0.293 \\
$C_{m x}$ & 0.0288 & 0.0507 & 0.0566 & 0.0593 & 0.0619 \\
$C_{d p}$ & 0.0306 & 0.0308 & 0.0285 & 0.0273 & 0.0262 \\
\hline
\end{tabular}

The global effect of the momentum coefficient on the separated flow can be better understood using the integral parameters given in Table 2. In this table (also in the future tables), the $C_{m x}$ and $C_{d p}$ coefficients include the fourth digits because of their one order of lower magnitudes compared to $C_{n p}$. Overall, the integral parameters are well correlated with the effect of momentum coefficient. The modified normal force $\left(C_{n p}\right)$ and the moment coefficients $\left(C_{m x}\right)$ increase, whereas the pressure drag coefficient decreases with the momentum coefficient. Note that initially the pressure drag coefficient increases slightly from the baseline case to the low $C_{\mu}(0.11 \%)$ excitation case. Negligible difference or slight increase in the pressure drag for a low $C_{\mu}$ excitation is a known fact. ${ }^{4,6}$ Although flow separation is reduced, the negligible difference or slight increase in the pressure drag coefficient may be attributed to the increase in the drag due to suction pressures (between $0.5<x / c<x_{m}$ ) that is similar to the drag-due-to-lift in airfoil applications.

The effect of momentum coefficient was also studied using the numerical simulations. Figure 7 shows the simulated pressure distributions compared to the experiments. The numerical and experimental $C_{p}$ distributions for the baseline case are also included in the figure for comparison. The momentum coefficients studied are $0.11 \%$ and $0.24 \%$. All pressure distributions are in good agreement upstream of the hump as well as in the favorable pressure gradient region. The pressure distributions of the flow control cases gradually deviate from those of the baseline cases in the pressure relaxation region $(x / c>0.2)$ where the suction pressures are higher. The experimental and numerical $C_{p}$ distributions of the flow control cases agree well in this region until the model midchord $(x / c=0.5)$. Despite the presence of the mild adverse pressure gradient, the flow keeps accelerating in the experiments until the suction peak at $x / c=0.61$. However, the numerical results are more sensitive to the adverse pressure gradient causing immediate flow deceleration. The pressure recovery due to the flow control is evident in both experimental and numerical results. Although the $C_{p}$ distributions of the numerical simulations and experiments do not agree well due to their baselines being different, the pressure recoveries relative to their baseline results are comparable. Steady excitations resulted in lower pressures (i.e., flow acceleration) immediately downstream of the nozzle exits compared to the baseline cases.

Table 3 compares the integral parameters of the numerical and experimental results for two different momentum coefficients. As presented in the $C_{p}$ distributions (Fig. 7), the $C_{\mu}=0.24 \%$ case obtained by experiment is the best control case whereas the simulated baseline case is the worst in terms of $C_{n p}$ and $C_{m x}$ coefficients. Although the magnitudes of the integral parameters of the numerical and experimental results do not agree well, they have similar trends. For example, $C_{n p}$ increased almost $50 \%$ from baseline to the low amplitude excitation $\left(C_{\mu}=0.11 \%\right)$ and $16 \%$ from the low amplitude $\left(C_{\mu}=0.11 \%\right)$ to high amplitude $\left(C_{\mu}=0.24 \%\right)$ excitations in the experiment whereas its relative

8

American Institute of Aeronautics and Astronautics 
increments in the numerical simulations are $48 \%$ and $14 \%$, respectively. As mentioned before, the pressure drag coefficient initially increases for low $C_{\mu}$ excitations and then gradually decreases as observed in both numerical and experimental results. Note that since the baseline separation bubble is longer in the numerical simulations, the pressure drag value is smaller but both the experiment and simulation have the same trend.

Table 3. Integral parameters of the numerical and experimental results for steady excitation.

\begin{tabular}{ccccccc}
\hline \hline & $\begin{array}{c}\text { Baseline } \\
\text { Exp }\end{array}$ & $\begin{array}{c}C_{\mu}=0.11 \% \\
\operatorname{Exp}\end{array}$ & $\begin{array}{c}C_{\mu}=0.24 \% \\
\operatorname{Exp}\end{array}$ & $\begin{array}{c}\text { Baseline } \\
\text { CFD }\end{array}$ & $\begin{array}{c}C_{\mu}=0.11 \% \\
\text { CFD }\end{array}$ & $\begin{array}{c}C_{\mu}=0.24 \% \\
\text { CFD }\end{array}$ \\
\hline$C_{n p}$ & 0.161 & 0.243 & 0.281 & 0.118 & 0.174 & 0.199 \\
$C_{m x}$ & 0.0288 & 0.0507 & 0.0593 & 0.0070 & 0.0331 & 0.0397 \\
$C_{d p}$ & 0.0306 & 0.0308 & 0.0273 & 0.0225 & 0.0328 & 0.0326 \\
\hline \hline
\end{tabular}

The time averaged spanwise vorticity contours with superimposed streamlines at the centerline clearly show the separated shear layer (Fig. 8). The flow control with steady excitation is not able to keep the flow attached and the shear layer is separated from the hump surface. At this particular location (centerline), the variations in the flow separation location is negligible $\left(x_{s}=0.665, x_{s}=0.667, x_{s}=0.670\right.$, for the baseline, $C_{\mu}=0.11 \%, C_{\mu}=0.24 \%$ cases, respectively). The effect of steady excitation is more pronounced at downstream locations as evident in the diffused shear layer. Compared to the baseline case (Fig. 8a), the high levels of vorticity extend over a shorter distance, which indicates a shorter separation bubble as shown by the streamlines. Surprisingly, increasing the momentum coefficient does not appear to reduce the extent of the shear layer. This is due to a local effect where the $C_{\mu}=0.11 \%$ case generates a locally attached flow near the exit of the centerline actuator (see Fig. 9). Spanwise vorticity contours at an off center location show a similar proportional effect of the momentum coefficient on the shear layer (not shown here). Although the difference is small, flow reattachment locations clearly show the reduced separation bubble for the higher amplitude case, where the reattachment locations are predicted as $x_{r}=1.266, x_{r}=1.050$ and $x_{r}=1.006$ for the baseline, $C_{\mu}=0.11 \%$ and $C_{\mu}=0.24 \%$ cases, respectively.

The three-dimensional extent of the separated flow and its control with the steady excitation is illustrated by the zero-streamwise-velocity isosurfaces in Fig. 9. In this figure, the flow direction is from top to bottom and only the separated flow region $(0.65<x / c<1.3)$ is shown. The isosurfaces of three cases (baseline, $C_{\mu}=0.11 \%$ and $C_{\mu}=$ $0.24 \%$ ) are superimposed to better demonstrate the effect of momentum coefficient on the separation bubble. As shown in this figure, the separation bubble is three dimensional. There are symmetric patterns of locally separated/attached flow regions for the flow control cases. These local regions are especially noticeable for higher amplitude excitations. It was reported in Ref. 13 that these patterns are due to the discrete jets and the generated streamwise vortices interacting with each other to form groups under the effect of the adverse pressure gradient. For example, a group of jets gradually merges and creates islands of attached flow. The steady jets on each side of the group bend inward causing gaps between the groups where the local flow is not affected as much by the flow control. As a result, these areas have larger separation bubbles. As consistent with the $C_{p}$ distribution and the spanwise vorticity contours, the steady excitation reduces the separation bubble size. Although not perfectly two-dimensional, the flow control with steady excitation induces more uniform flow reattachment along the span compared to the baseline case.

\section{B. Flow Control with Unsteady Excitation}

The same nozzle/valve/plenum configuration was used to generate the unsteady excitation. In the experiments, a square wave trigger signal was sent to the high-speed valves to generate pulsed-type excitation and the mass flow rate to the plenum was adjusted based on the required momentum coefficient. The duty cycle is the same (50\%) for all unsteady excitation cases. In the numerical simulations, an unsteady (square wave type) mass flow boundary condition was prescribed at each nozzle inlet (see inset in Fig. 1).

Instantaneous vortical structures generated by the unsteady excitation are presented in Fig. 10. This figure shows instantaneous isosurfaces of $\lambda_{2}$ - criterion colored by the streamwise velocity to highlight the vortex cores. For this particular case, the momentum coefficient and the excitation frequency are $0.24 \%, 135 \mathrm{~Hz}$, respectively. This frequency was chosen based on the previous study that used the ZNMF actuators. The reduced frequency based on the reattachment location is slightly larger ( 0.82 vs. 0.77 in Workshop) due to the reattachment location being slightly different from that of the Workshop ( $x_{r}=1.15$ vs. $\left.x_{r}=1.11\right)$. Consecutive vortical structures due to the unsteady excitation are clearly seen. Although the excitation was introduced from discrete nozzles, the convected vortical 
structures indicate that the effect of excitation is fairly uniform across the span. When compared to the steady excitation case at the same momentum coefficient (Fig. 5), one can notice different vortical structures due to the unsteady excitation. While the steady excitation resulted in the formation of a wide variety of three-dimensional turbulent structures especially aft of the model, the vortical structures are more organized in the unsteady excitation case. In the near exit regions, only smaller scale vortical structures are present and they start to grow downstream of the trailing edge $(x / c>1)$.

The effect of excitation frequency on the centerline pressure distributions was studied first in the wind tunnel experiments (Fig. 11). For this particular case, the momentum coefficient is $0.24 \%$. The pressure distributions of the baseline and the steady excitation are included for comparison. Upstream of the separation, the $C_{p}$ distribution of the low frequency $(10 \mathrm{~Hz})$ excitation closely follows that of the steady excitation. Increasing the excitation frequency provided slightly better suction pressures but no noticeable difference between mid $(60 \mathrm{~Hz})$ and high $(135 \mathrm{~Hz})$ frequency excitations. Pressure recovery characteristics downstream of flow separation show distinct features. First, the offset in the pressure distributions between the steady and unsteady excitations clearly demonstrates the superiority of the unsteady excitation. Secondly, it is found that unsteady excitation is especially effective in the near excitation regions $(x / c \approx 0.7)$. As previously shown in Fig. 6 , even increasing the excitation amplitude was not able to increase the pressure recovery in the near exit regions due to the severe adverse pressure gradient. The excitation with the lowest frequency is able to provide better pressure recovery than the steady excitation in this region. This may be due to the more organized structures that were shown in Fig. 10 produced by unsteady excitation. Comparing Fig. 5 and Fig. 10, the unsteady excitation suppresses the large-scale structures in the near exit regions and delays their formation farther downstream. Increasing the excitation frequency does not increase the pressure recovery in the near field, and all frequency excitations provided similar pressure recovery in the immediate downstream regions. The effect of frequency is more pronounced at downstream locations (close to the hump trailing edge) where increasing the excitation frequency provided more pressure recovery. Overall, the unsteady excitation provides increased suction pressures upstream and better pressure recovery downstream. The higher frequencies provided better flow control authority at this frequency range. Note that the highest excitation frequency $(135 \mathrm{~Hz})$ tested corresponds to the nondimensional frequency of 0.82 , where the optimal nondimensional frequency was reported to be around $1.5 .^{4}$ Higher frequencies could not be tested due to experimental limitations. The effect of frequency was also studied using numerical simulations. However, negligible differences were observed between the $60 \mathrm{~Hz}$ and $135 \mathrm{~Hz}$ cases, both in the simulated $C_{p}$ distributions as well as in the separation bubble and therefore were not reported here.

Integral parameters also support the conclusions stated above. As tabulated in Table 4, the modified normal force and moment coefficients gradually increase with the excitation frequency. $C_{n p}$ and $C_{m x}$ for the steady excitation are close to the low frequency unsteady excitation but for other frequencies, the unsteady excitation is superior to the steady excitation. The pressure drag coefficient appears to increase slightly with the increased excitation frequency. Since the higher frequencies produce higher suction pressures upstream and better pressure recoveries downstream, the slight increase in the pressure drag coefficient might be due to the increase in the suction pressure drag (i.e., dragdue-to-lift) between $0.5<x / c<x_{m}$. Although, the pressure drag coefficient is increasing with the excitation frequency, all of the tested frequencies produce lower drag coefficient than the steady excitation case.

Table 4. Variation of integral parameters with respect to excitation frequency.

\begin{tabular}{cccccccc}
\hline \hline & Baseline & Steady & $5 \mathrm{~Hz}$ & $10 \mathrm{~Hz}$ & $20 \mathrm{~Hz}$ & $60 \mathrm{~Hz}$ & $135 \mathrm{~Hz}$ \\
\hline$C_{n p}$ & 0.161 & 0.281 & 0.278 & 0.286 & 0.292 & 0.310 & 0.314 \\
$C_{m x}$ & 0.0288 & 0.0593 & 0.0564 & 0.0593 & 0.0617 & 0.0670 & 0.0675 \\
$C_{d p}$ & 0.0306 & 0.0273 & 0.0197 & 0.0205 & 0.0223 & 0.0229 & 0.0232 \\
\hline \hline
\end{tabular}

The effect of momentum coefficient on the centerline pressure distributions for the unsteady excitations is given in Fig. 12. The oscillations in the numerical $C_{p}$ distributions near the actuator exit are due to the high momentum jet excitation and the magnitudes of the oscillations increase as the momentum coefficient increases. The same conclusions made for the steady excitation case (Fig. 7) can be made for this case as well. The numerical simulations predicted lower suction pressures and less pressure recovery compared to the experiment. The differences between the numerical simulations and experiment are more pronounced as the momentum coefficient increases. As one can expect, increasing the momentum coefficient provides better flow control authority. Compared to the steady excitation case (Fig. 7), it is clear that the unsteady excitation is more beneficial as indicated by the increased suction pressures upstream and pressure recovery downstream. The relative pressure recoveries (offset between the low and high 
momentum excitations in Fig. 12) also appear to be larger than those of the steady excitation cases presented in Fig. 7. Numerical simulations also predicted the increased effectiveness of the unsteady excitations as shown by the spanwise vorticity contours (Fig. 13). This level of unsteady excitation, again, is not enough to keep the flow attached, and the shear layer is shown to be separated for all cases. However, it is clearly seen that the region of highest vorticity shortens and gets closer to the hump surface as the momentum coefficient is increased. Slight separation delay is also observed at the centerline $\left(x_{s}=0.665, x_{s}=0.677\right.$ and $x_{s}=0.678$ for the baseline, $C_{\mu}=0.11 \%$ and $C_{\mu}=0.24 \%$ cases, respectively) possibly due to the local effects induced by the high momentum blowing. Superimposed streamlines show the separation bubbles for the three cases. The reattachment locations are predicted to be $x_{r}=1.266, x_{r}=1.066$ and $x_{r}=1.022$ for the baseline, $C_{\mu}=0.11 \%$ and $C_{\mu}=0.24 \%$ cases, respectively. The separation bubble at the centerline is found to be slightly larger $\left(x_{r}=1.066\right.$ vs. 1.050 and $x_{r}=1.022$ vs. 1.006) than that of the steady excitation cases. However, these differences are merely due to the nonuniform reattachment lines and cannot be generalized as shown in the zero-streamwise-velocity isosurfaces (Fig. 14). Flow separation appears to be more uniform than the steady excitation case (in Fig. 9), as the local separated/attached flow regions downstream of the nozzle exits are substantially reduced. The effect of momentum coefficient on the separation bubble is also apparent in the figure generating a smaller separation bubble across the span.

The overall effect of the unsteady excitation can be better explained using the integral parameters. As given in Table 5, the modified normal force and moment coefficients correlate well with the momentum coefficient. These integral parameters increase gradually with the momentum coefficient for both numerical and experimental results. The rate of increase of these parameters indicates that the unsteady excitation is more effective than the steady excitation (given in Table 3). The pressure drag coefficient calculated using the experimental $C_{p}$ distribution shows the favorable effect of the unsteady excitation on the pressure drag. Again, $C_{d p}$ is more sensitive to the unsteady excitation such that even the low $C_{\mu}$ excitation generates a noticeable reduction in the pressure drag. $C_{d p}$ predicted by numerical simulations shows similar behavior to the steady excitation (i.e., $C_{d p}$ increases at low $C_{\mu}$ excitations); however, comparing the drag coefficient values with that of the steady excitations clearly indicates superiority of the unsteady excitation.

Table 5. Comparison of integral parameters for different unsteady excitation amplitudes.

\begin{tabular}{ccccccc}
\hline \hline & $\begin{array}{c}\text { Baseline } \\
\operatorname{Exp}\end{array}$ & $\begin{array}{c}C_{\mu}=0.11 \% \\
\operatorname{Exp}\end{array}$ & $\begin{array}{c}C_{\mu}=0.24 \% \\
\operatorname{Exp}\end{array}$ & $\begin{array}{c}\text { Baseline } \\
\text { CFD }\end{array}$ & $\begin{array}{c}C_{\mu}=0.11 \% \\
\text { CFD }\end{array}$ & $\begin{array}{c}C_{\mu}=0.24 \% \\
\text { CFD }\end{array}$ \\
\hline$C_{n p}$ & 0.161 & 0.257 & 0.314 & 0.118 & 0.185 & 0.234 \\
$C_{m x}$ & 0.0288 & 0.0546 & 0.0675 & 0.0070 & 0.0367 & 0.0503 \\
$C_{d p}$ & 0.0306 & 0.0279 & 0.0232 & 0.0225 & 0.0316 & 0.0311 \\
\hline \hline
\end{tabular}

\section{Performance Evaluation of Flow Control Methods}

Although the wall-mounted hump model is a benchmark case for CFD applications, various flow control methods have also been tested on this model. In this section, the performance of these different flow control methods will be assessed using the integral parameters defined in this study. With the exception of the attached flow case, the performance of the flow control methods were evaluated at moderate $C_{\mu}$ values $\left(C_{\mu}=0.11 \%\right.$ and $\left.C_{\mu}=0.24 \%\right)$. As indicated by Greenblatt et al., ${ }^{4}$ these $C_{\mu}$ values were chosen such that the flow control methods exert substantial control over the separation region without entirely eliminating the bubble for successful comparison of the different flow control methods. All of the actuators were placed at the $65 \%$ of the hump chord $(x / c=0.65)$ with the exception of the VGs, which were placed at $x / c=0.5$ (i.e., the suction peak location in the baseline case) to generate stronger streamwise vortices. The experimental configurations are also the same $\left(\operatorname{Re}_{c}=0.4 \times 10^{6}\right)$ with the exception of the attached flow case, which was tested in a high Reynolds number experiment.

Figure 15 shows the centerline pressure distribution over the hump model for different flow control techniques. The figure also includes the $C_{p}$ distributions of the separated and inviscid flow over the hump model at the same conditions. Being an ideal case, inviscid flow over the hump model produces suction pressures over most of the surface and quick pressure recovery as it was designed. The attached flow case shown in this figure was reported by Seifert et al..$^{22}$ at high Reynolds numbers. The Mach number was 0.25 , which corresponded to $R e_{c}=16 \times 10^{6}$. The pressure distribution was adjusted to match the inflow $C_{p}$ distribution as suggested by Greenblatt et al. ${ }^{3-4}$ The attached flow case was obtained using a steady blowing from a two-dimensional slot with the momentum coefficient of $1.68 \%$. 
The state of the attached flow was confirmed by checking the fluctuating pressures (see Fig. 19 in Ref. 22). As shown in this image, the $C_{p}$ distribution of the attached flow case is similar to the baseline case until the flow relaxation region $(x / c<0.2)$, later it deviates from the baseline case and shows comparable pressure levels as the inviscid flow. The trend of the downstream pressure recovery is also similar to the inviscid case with a slight offset in the pressure values. Flow control by passive vortex generators (VG) was reported in Ref. 14. There were 17 counter rotating VG pairs installed near the model midchord (where the baseline case has a suction peak). The spacing of the VG pairs $(16.5 \mathrm{~mm})$ was double the spacing of the current jet nozzle. The suction peak near the model midchord is due to the local flow acceleration between two VGs. The second suction peak was observed slightly upstream of that of the inviscid case possibly due to the missing pressure ports near this location. The passive VGs also provided substantial pressure recovery compared to the baseline case; however, this occurs over a longer distance compared to the ideal flow case. The separation bubble was observed in the oilflow visualization and the flow reattachment was reported as $x_{r}=0.75{ }^{14}$

The integral parameters for these different flow control methods are given in Table 6. As an ideal case, the inviscid flow provided the highest $C_{n p}$ and $C_{m x}$ values and generated net thrust. The attached flow case with steady blowing $\left(C_{\mu}=1.68 \%\right)$ increases the $C_{n p}$ and $C_{m x}$ coefficients more than two times compared to the baseline case. Attached flow produced an almost zero pressure drag coefficient. Although the flow is attached, it could not produce thrust on this model. This is because the suction pressures especially in the first half of the model $(x / c<0.5)$ are low compared to the inviscid case in the previous figure. The passive flow control with VGs also increased the $C_{n p}$ and $C_{m x}$ coefficients almost two times compared to the baseline case. Although the pressure drag coefficient is reduced by $25 \%$ compared to the baseline case, it is considerably higher than that of the attached flow case due to the separation bubble observed in the domain (see Fig. 11 in Ref. 14).

Table 6. Comparison of different flow configurations.

\begin{tabular}{ccccc}
\hline \hline & Baseline Flow & Inviscid Flow & Attached Flow & VG Control \\
\hline$C_{n p}$ & 0.161 & 0.506 & 0.386 & 0.306 \\
$C_{m x}$ & 0.0288 & 0.1052 & 0.0680 & 0.0598 \\
$C_{d p}$ & 0.0306 & -0.0133 & 0.0008 & 0.0234 \\
\hline \hline
\end{tabular}

Figure 16 shows the comparison of different active flow control (AFC) methods used to control flow separation over the hump model for low amplitude excitation $\left(C_{\mu}=0.11 \%\right)$. The ZNMF actuators were reported by Greenblatt et $\mathrm{al}^{4}{ }^{4}$ in the CFDVAL2004 Workshop. The temporally oscillating jet was introduced from the two-dimensional spanwise slot $(0.75 \mathrm{~mm}$ by $584 \mathrm{~mm})$ using voice-coil based actuators. The frequency of the ZNMF actuators for this particular case was $138.5 \mathrm{~Hz}$. The SWJ actuator study was reported in Ref. 14 where the spatially oscillating jet was introduced from $17 \mathrm{SWJ}$ actuators (1 $\mathrm{mm}$ by $2 \mathrm{~mm}$ throat) with $33 \mathrm{~mm}$ spacing. The frequency of the SWJ actuators was reported as $550 \mathrm{~Hz}$ for this particular momentum coefficient. Steady and unsteady (i.e., pulsed) jets are introduced from 31 discrete nozzles $(1 \mathrm{~mm}$ by $5.6 \mathrm{~mm}$ ) with $16.5 \mathrm{~mm}$ spacing that are reported in this study. The frequency of the unsteady excitation is $135 \mathrm{~Hz}$. As shown in this figure, all of the actuators increase the suction pressure upstream and the pressure recovery downstream compared to the baseline case. Out of all actuators, the ZNMF actuators appear to be the least effective. The rest of the actuators (SWJ, Steady, Pulsed) produced similar suction pressure upstream. In the pressure recovery region, the superiority of the unsteady excitation is observed in the form of better pressure recovery, although the SWJ and steady actuators produced a similar level of pressure recovery.

These conclusions are confirmed by the integral parameters given in Table 7. By looking at the $C_{n p}$ and $C_{m x}$ coefficients, the unsteady excitation (pulsed jet) was found to be the most effective, closely followed by the SWJ actuators and then the steady excitation. The ZNMF was found to be the least effective. As shown in this table, the ZNMF actuators produced even higher-pressure drag coefficient than the baseline case. Although the separation bubble was reported to be reduced $\left(x_{r}=0.98\right),{ }^{4}$ the pressure recovery immediately downstream of the actuators is not improved. Possibly, the ZNMF actuators amplified the circulation of the separation bubble and generated even higher levels of suction pressure over the separation bubble. In this region $\left(x_{m}<x / c<0.8\right)$, the pressure gradient is severe and contributes most to the pressure drag coefficient; therefore, higher pressure drag values are observed compared to the baseline case. Again, the application of unsteady excitation provided the least pressure drag, followed by the SWJ actuators. 
Table 7. Different AFC methods at low excitation amplitude $\left(C_{\mu}=\mathbf{0 . 1 1 \%}\right)$

\begin{tabular}{cccccc}
\hline \hline & Baseline & ZNMF & SWJ & Steady & Pulsed \\
\hline$C_{n p}$ & 0.161 & 0.211 & 0.255 & 0.243 & 0.257 \\
$C_{m x}$ & 0.0288 & 0.0440 & 0.0540 & 0.0507 & 0.0546 \\
$C_{d p}$ & 0.0306 & 0.0336 & 0.0291 & 0.0308 & 0.0279 \\
\hline \hline
\end{tabular}

Figure 17 presents the comparison of different AFC methods for a higher excitation amplitude $\left(C_{\mu}=0.24 \%\right)$. The suction was introduced from the same two-dimensional slot using a vacuum pump that was attached to the plenum and reported by Greenblatt et al. ${ }^{3}$ in the CFDVAL2004 Workshop. As expected, all AFC methods provide better flow control authority as the momentum coefficient is increased. The actuators provide higher suction pressures upstream and more pressure recovery downstream. The steady excitation appears to be the least effective by looking at the $C_{p}$ distributions. On the other hand, the suction method generates higher suction pressures immediately upstream of the slot. The SWJ and unsteady excitations provide similar suction pressures to the suction method with the exception of two missing pressure ports where the suction method has a peak. In the pressure recovery region, each actuator has different characteristics. The suction method provides substantial pressure drop immediately downstream of the slot but this drop does not persist further downstream. The $C_{p}$ distribution has a plateau (similar to the baseline case), which indicates a possible separation bubble. In fact, Greenblatt et al. ${ }^{3}$ reported a flow separation bubble where the flow reattachment was at $x_{r}=0.94$. After the plateau, the pressure recovery curve is very close to the steady excitation, which provides the least pressure recovery. The unsteady excitation produces the lowest pressure drop in the pressure ports closest to the actuator exits $(x / c=0.687)$; however, the pressure drops faster and the unsteady excitation generates the best pressure recovery after $x / c>0.73$. The SWJ actuators produce a $C_{p}$ distribution that is closest to the unsteady excitation.

The integral parameters given in Table 8 will be helpful for overall comparison of the AFC actuators at a higher excitation amplitude. As a well-known fact, the suction method provides the best control by reducing the pressure drag coefficient by $50 \%$ and doubling the normal force and moment coefficients. As consistent with the low amplitude case (Fig. $16 \&$ Table 7), the steady excitation appears to be the least effective out of four actuators as indicated by all integral parameters. The normal force coefficients of the SWJ and unsteady excitations appear to be lower than the suction method although their moment coefficients are similar. This is partly due to the two missing pressure ports that are immediately upstream of the actuator exits. This confirms that the moment coefficient is less sensitive to the local pressure variations due to the actuator itself. Considering the previously given uncertainty estimates, the unsteady excitation appears to be slightly more effective than the SWJ actuators as indicated by all three integral parameters although the numbers are close.

Table 8. Different AFC methods at high excitation amplitude $\left(C_{\mu}=0.24 \%\right)$.

\begin{tabular}{cccccc}
\hline \hline & Baseline & Suction & SWJ & SJ & PJ \\
\hline$C_{n p}$ & 0.161 & 0.333 & 0.313 & 0.281 & 0.314 \\
$C_{m x}$ & 0.0288 & 0.0662 & 0.0661 & 0.0593 & 0.0675 \\
$C_{d p}$ & 0.0306 & 0.0156 & 0.0235 & 0.0273 & 0.0232 \\
\hline \hline
\end{tabular}

\section{Conclusion}

Flow separation and its control with steady/unsteady excitation were investigated at subsonic speeds. The wall mounted hump model was selected as the test bed as the model has been well documented numerically and experimentally. Wind tunnel experiments and numerical simulations were carried out in parallel to understand the effect of the flow control on the separated flow. An array of discrete nozzles together with high speed valves were used as active flow control actuators to generate steady/unsteady excitations. A lattice Boltzmann method based software package was used to simulate the unsteady flow over the hump model. Numerical simulations included the three-dimensional hump geometry, endplates, splitter plate, and the jet nozzles. Several integral parameters were explored to evaluate different flow configurations. The performance evaluation of different flow control methods including the current and previously tested methods was performed using the integral parameters. 
First, the baseline-separated flow was considered. Overall, the centerline pressure distribution obtained by the numerical simulations agreed well with the experiment with the exception of the separated flow region. Threedimensional numerical simulations could not accurately predict the separated flow resulting in a longer separation bubble compared to the experiment. The nondimensional reattachment location near the centerline was predicted to be 1.266 , but was reported as 1.15 in the experiments. Although the flow over the hump model was reported as nominally two-dimensional in the experiments, the separated flow region was found to be three-dimensional in the simulations.

Several integral parameters based on the pressure distributions were explored for the baseline case. Inviscid flow solution over the hump model was also included to evaluate these parameters. The pressure drag coefficient, normal force coefficient, the moment coefficient (about the quarter-chord) and the inviscid comparison coefficient were the integral parameters that have been reported in the literature. In addition to these quality metrics, two new integral parameters were introduced to better capture the requirement that for a flow control method to be effective on the hump, it must produce higher suction pressures upstream of the separation and better pressure recovery downstream of it. In order to satisfy this requirement, the normal force and the moment coefficients were slightly modified. The sign of the first parameter was changed for the pressure recovery region aft of the hump model $\left(x>x_{m}\right)$. In the second parameter, the moment coefficient was based on this point of discontinuity instead of quarter-chord location. By definition, these two modifications provide a direct indication of higher suction pressure upstream and better pressure recovery downstream. These two integral parameters together with the pressure drag coefficient were found to be well-correlated with the effectiveness of the flow control method and were used as quality metrics in the performance evaluation of the flow control methods.

Steady excitation was applied as an active flow control (AFC) method to control flow separation over the hump model. By gradually increasing the flow rate to the actuators, the effect of momentum coefficient was studied both numerically and experimentally. As expected, better flow control authority was observed as the momentum coefficient was increased in the experiments. This is confirmed by all integral parameters with the exception of the pressure drag coefficient, which increased slightly for low amplitude excitations. The numerical simulations also showed the effectiveness of the steady excitation by reducing the separation bubble as well as inducing more uniform flow reattachment along the span compared to the baseline case.

Unsteady excitation was also applied to the hump model and the effects of frequency and amplitude were investigated both numerically and experimentally. Although the excitations were introduced from discrete nozzles, the simulated vortical structures indicated that the effect of the unsteady excitation is fairly uniform across the span. It was found that the unsteady excitation was very effective in controlling flow separation, especially in the pressure recovery region near the separation location where the adverse pressure gradient is severe and the steady excitation was not able to provide sufficient control. This favorable effect of the unsteady excitation was attributed to the more organized structures and the suppression of the growth of the large-scale structures in this region. This is an important finding, as the flow control at severe adverse pressure gradients (such as high flap deflection) is known to be challenging. The effect of momentum coefficient was similar to that of steady excitations where higher amplitude excitations provide better flow control authority. On the other hand, the effect of excitation frequency was more pronounced near the flow reattachment regions. Although the pressure distributions and hence the integral parameters predicted by numerical simulations usually indicate less flow control authority compared to the experiments, they show a similar trend.

As part of this study, a performance evaluation of different flow control methods was performed by comparing the pressure distributions and their integral parameters. First, the baseline case, inviscid flow, attached flow with steady blowing and vortex generators were compared. As an ideal case, inviscid flow produced high suction pressure upstream and good pressure recovery downstream without any flow separation. Inviscid flow produced net thrust on this model and as expected, the integral parameters indicated that this was the best flow configuration. The attached flow with steady blowing $\left(C_{\mu}=1.68 \%\right)$ generated almost zero pressure drag coefficient and increased the modified force and moment coefficients more than two times compared to the baseline case. Although the VG flow control also increased the modified force and moment coefficients, the pressure drag coefficient was only reduced by $25 \%$ due to the existing separation bubble. Secondly, the AFC methods were compared at low amplitude excitations $\left(C_{\mu}\right.$ $=0.11 \%$ ). Results of zero net mass flux (ZNMF) actuators, sweeping jet (SWJ) actuators from previous studies were compared to the currently studied steady and unsteady excitations. Although all AFC methods increased the upstream suction pressure and pressure recovery downstream, the ZNMF actuators were found to be the least effective at this configuration. The ZNMF actuators generated an even higher-pressure drag coefficient although the separation bubble was reported to be reduced. The current unsteady excitation was found to be the most effective AFC method, closely followed by the SWJ actuators. The AFC methods were also compared at slightly higher amplitudes $\left(C_{\mu}=0.24 \%\right)$. 
Steady suction and SWJ actuators were also compared to the currently studied steady and unsteady excitations. As expected, steady suction provided the best control. The pressure drag coefficient was reduced and the integral parameters were doubled in value. According to the integral parameters, steady excitation was the least effective out of the four actuators. Although close, the unsteady excitation appears to be slightly more effective than the SWJ actuators in controlling flow separation over the hump model.

\section{Acknowledgements}

The author would like to thank the NASA Advanced Air Transport Technology Project for funding this research and the following individuals for their support: Catherine McGinley, Luther Jenkins, Latunia Melton, John Lin, and Charlie Debro. The author acknowledges the support provided by Benjamin Duda of Exa Corp.

\section{References}

${ }^{1}$ Glauert, M. B., “The Design of Suction Aerofoils with a Very Large CL-Range,” Aeronautical Research Council, R\&M 2111, Nov. 1945.

${ }^{2}$ Glauert, M. B., Walker,W. S., Raymer, W. G., and Gregory, N., "Wind-Tunnel Tests on a Thick Suction Aerofoil with a Single Slot," Aeronautical Research Council, R\&M 2646, Oct. 1948.

${ }^{3}$ Greenblatt, D., Paschal, K. B., Yao, C. S., Harris, J., Schaeffler, N. W., and Washburn, A. E., "Experimental Investigation of Separation Control Part 1: Baseline and Steady Suction," AIAA Journal, Vol. 44, No. 12, 2006, pp. 2820-2830.

${ }^{4}$ Greenblatt, D., Paschal, K., Yao, C.-S., and Harris, J., "Experimental Investigation of Separation Control Part 2: Zero MassEfflux Oscillatory Blowing," AIAA Journal, Vol. 44, No. 12, 2006b, pp. 2831-2845.

${ }^{5}$ Naughton, J. W., Viken, S., and Greenblatt, D., "Skin Friction Measurements on the NASA Hump Model," AIAA Journal, Vol. 44, No. 6, 2006, pp. 1255-1265.

${ }^{6}$ Seifert, A. and Pack, L. G., "Active Flow Separation Control on Wall-Mounted Hump at High Reynolds Numbers," AIAA Journal, Vol. 40, No. 7, 2002, pp. 1363-1372.

${ }^{7}$ Rumsey, C. L., Gatski, T, B., Sellers, W, L., Vatsa, V, N., and Viken, S. A., "Summary of the 2004 CFD Validation Workshop on Synthetic Jets and Turbulent Separation Control," AIAA Paper 2004-2217, 2004

${ }^{8}$ Goldschmied, F.R., "Fuselage Self-Propulsion by Static- Pressure Thrust: Wind-Tunnel Verification", AIAA paper 19872935, Sept. 1987.

${ }^{9}$ Franck, J. A., and Colonius, T., "Compressible Large-Eddy Simulation of Separation Control on a Wall-Mounted Hump," AIAA Journal, Vol. 48, No. 6, 2010, pp. 1098-1107.

${ }^{10}$ Morgan, P. E., Rizzetta, D. P., and Visbal, M. R., "Large-Eddy Simulation of Separation Control for Flow over a WallMounted Hump, “AIAA Journal, Vol. 45, No. 11, 2007, pp. 2643-2660.

${ }^{11}$ Yagiz, B., Guzel G., and Koc I., "Simulation of the Separated Flow over a Wall-Mounted Hump using Finite-Volume Based Lattice Boltzmann Method”, AIAA Paper 2015-3422, June 2015.

${ }^{12}$ Duda, B., and Fares, E., "Application of a Lattice-Boltzmann Method to the Separated Flow behind the NASA Hump," AIAA Paper 2016-1836, Jan. 2016.

${ }^{13}$ Koklu, M., "A Numerical and Experimental Investigation of Flow Separation Control over a Wall-Mounted Hump Model”, AIAA Paper 2018-1280, Jan. 2018.

${ }^{14}$ Koklu, M., "Application of Sweeping Jet Actuators on the NASA Hump Model and Comparison with CFDVAL2004 Experiments", AIAA Paper 2017-3313, June 2017.

${ }^{15}$ Borgmann, D., Pande, A., Little, J., and Woszidlo, R., "Experimental Study of Discrete Jet Forcing for Flow Separation Control on a Wall Mounted Hump", AIAA Paper 2017-1450, Jan. 2017.

${ }^{16}$ Kara, K., "Flow Separation Control using Sweeping Jet Actuator", AIAA Paper-3041, June 2017.

${ }^{17}$ Chen, S. and Doolen, G., "Lattice Boltzmann Method for Fluid Flows," Annual Review of Fluid Mechanics, Vol. 30, 1998, pp. 329-364.

${ }^{18}$ Teixeira, C., "Incorporating Turbulence Models into the Lattice-Boltzmann Method," International Journal of Modern Physics, Vol. 9, 1998, pp. 1159-1175.

${ }^{19}$ Seifert, A., "Evaluation Criteria and Performance Comparison of Actuators for Bluff-body Flow Control", AIAA-paper 20142400, June 2014.

${ }^{20}$ Yeh, C., Munday, P., Taira, K., and Munson, M. J., "Drag Reduction Control for Flow over a Hump with Surface-Mounted Thermoacoustic Actuator," AIAA Paper 2015-0826, 2015

${ }^{21}$ AGARD Working Group, "Standard: Assessment of Experimental Uncertainty With Application to Wind Tunnel Testing (AIAA S-071A-1999)", AIAA Standards.

${ }^{22}$ Seifert, A., and Pack, L. G., "Active Control of Separated Flows on Generic Configurations at High Reynolds Numbers", AIAA paper 1999-3403, June 1999. 


\section{FIGURES}

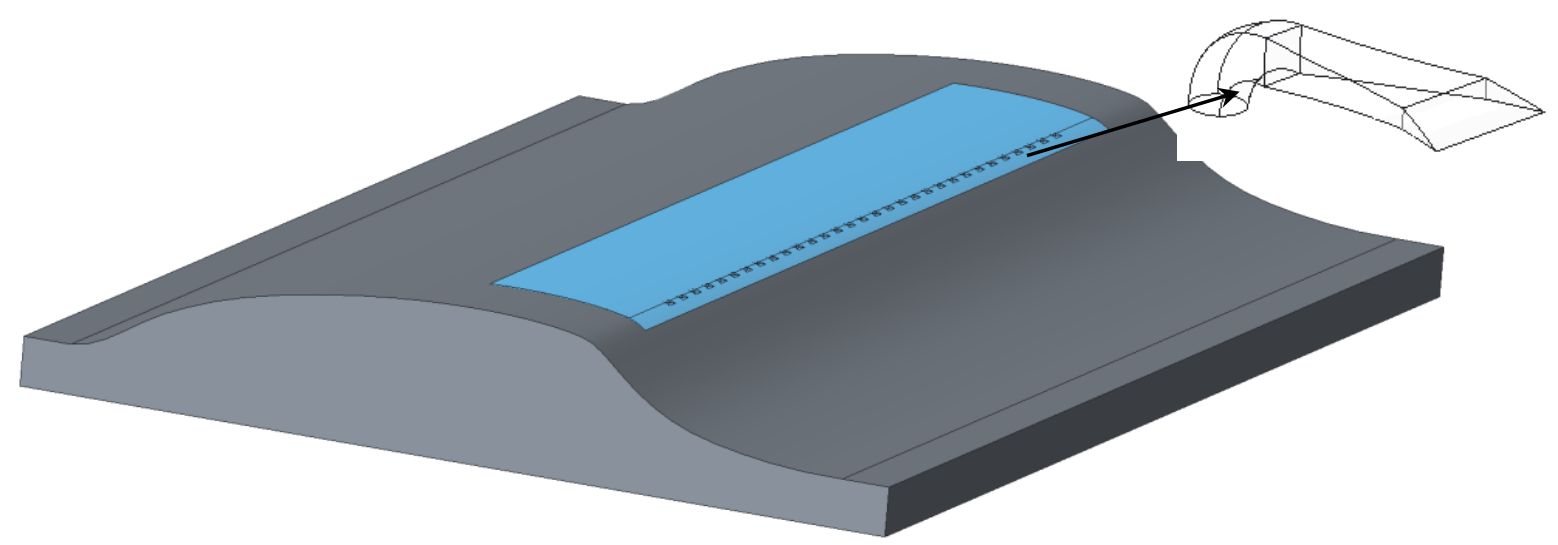

Figure 1. CAD rendering of the hump model without endplates (inset shows the jet nozzle geometry).

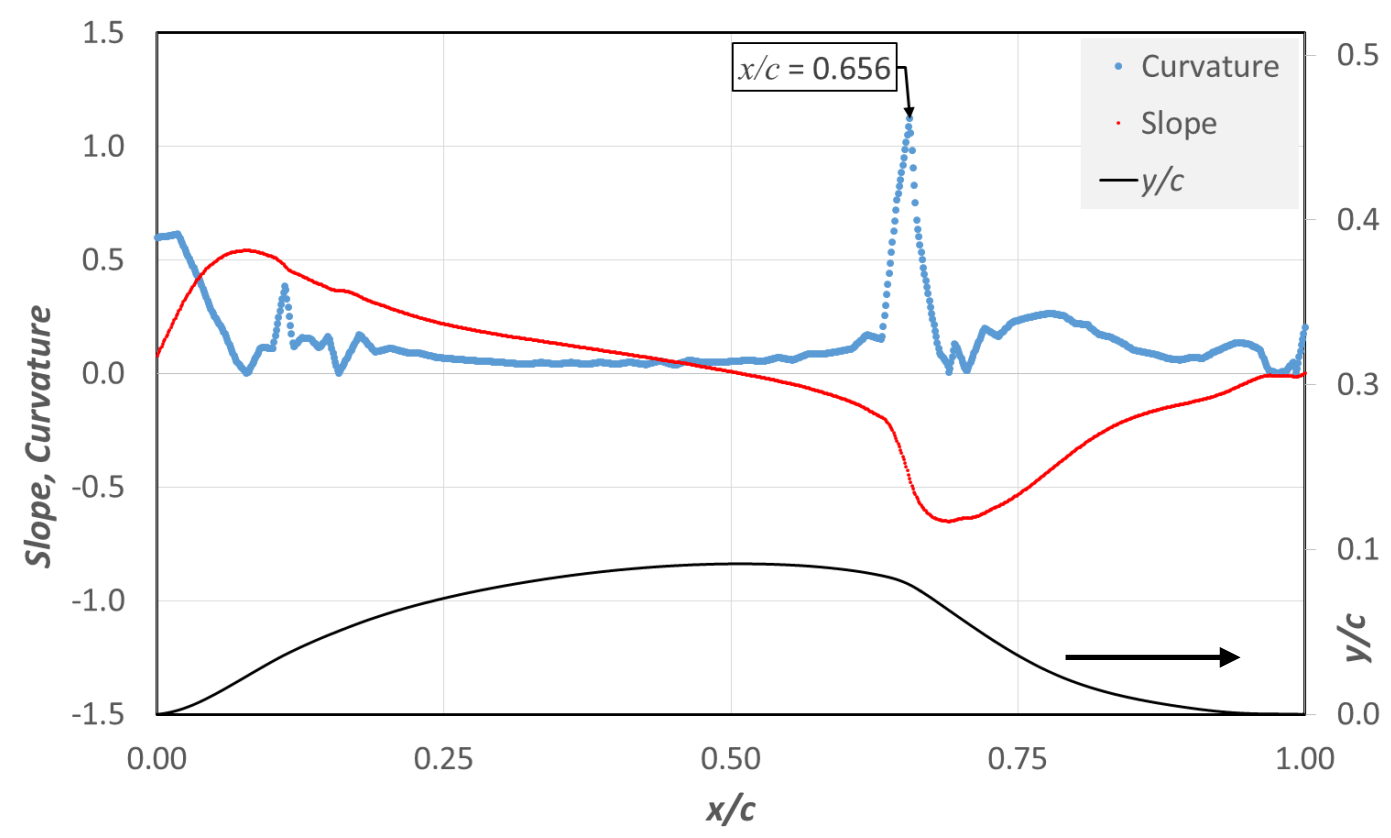

Figure 2. Slope and curvature of the wall-mounted hump model. 


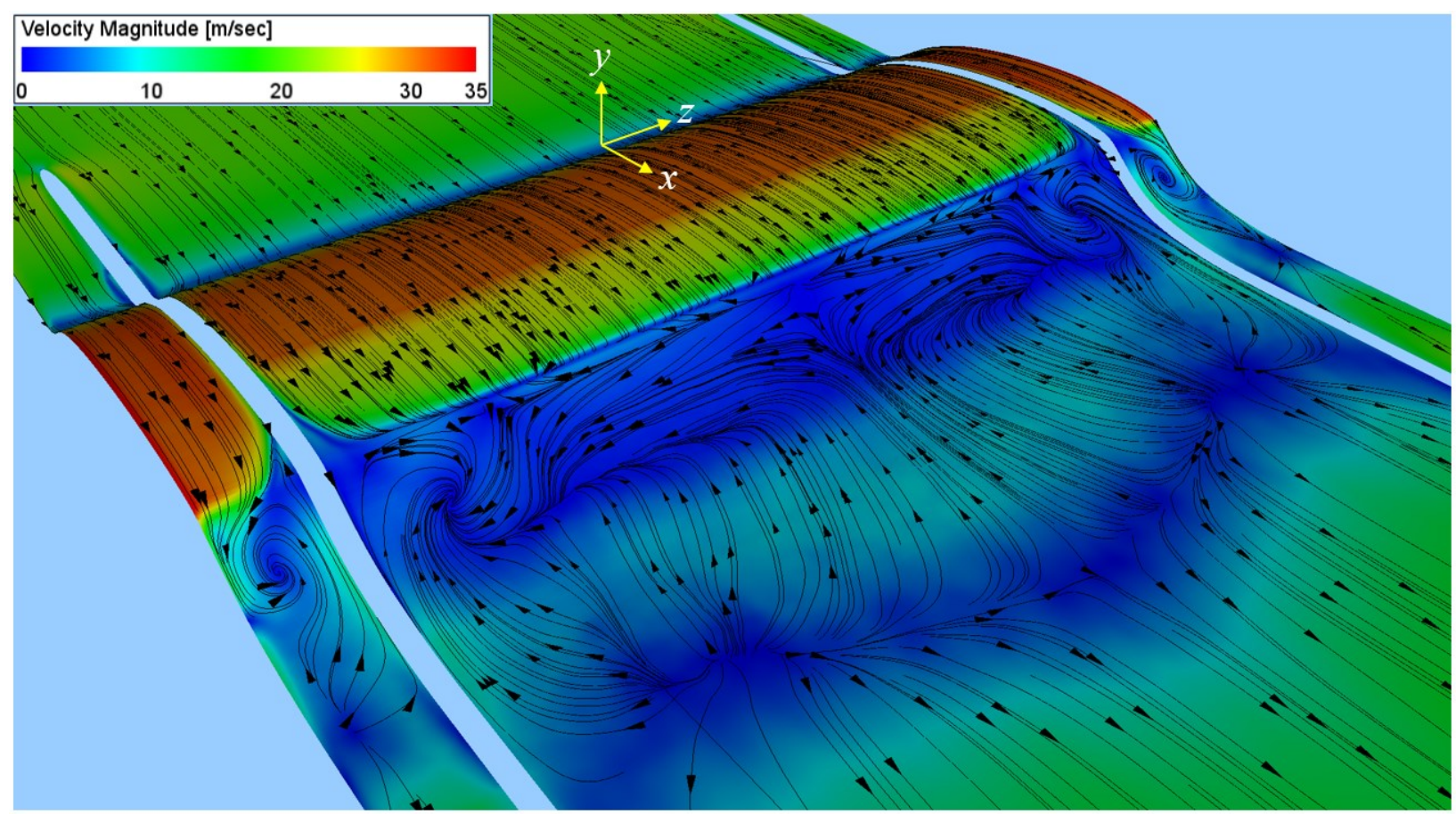

Figure 3. Surface flow visualization of the separated flow over the hump model.

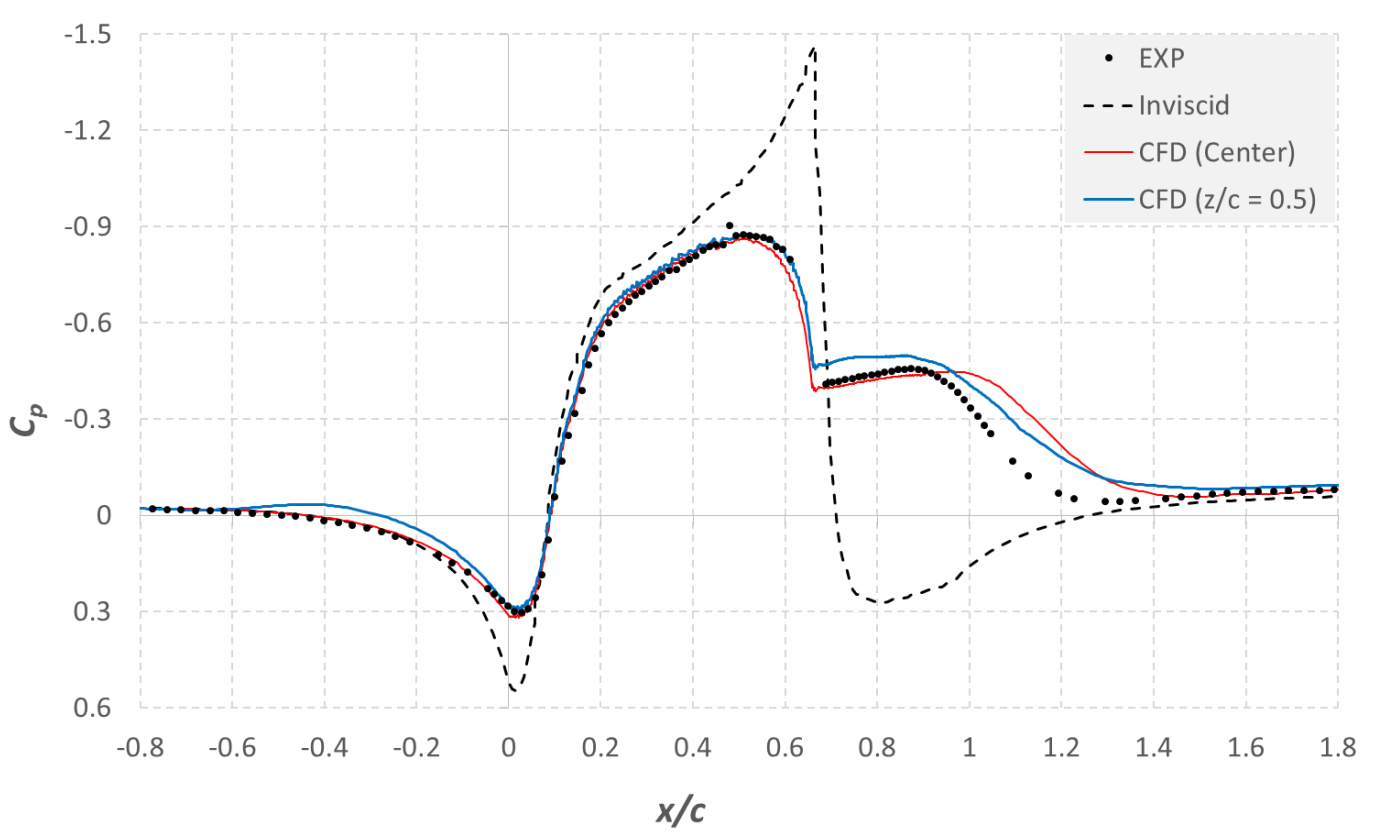

Figure 4. Numerical and experimental pressure distributions for the baseline case. 


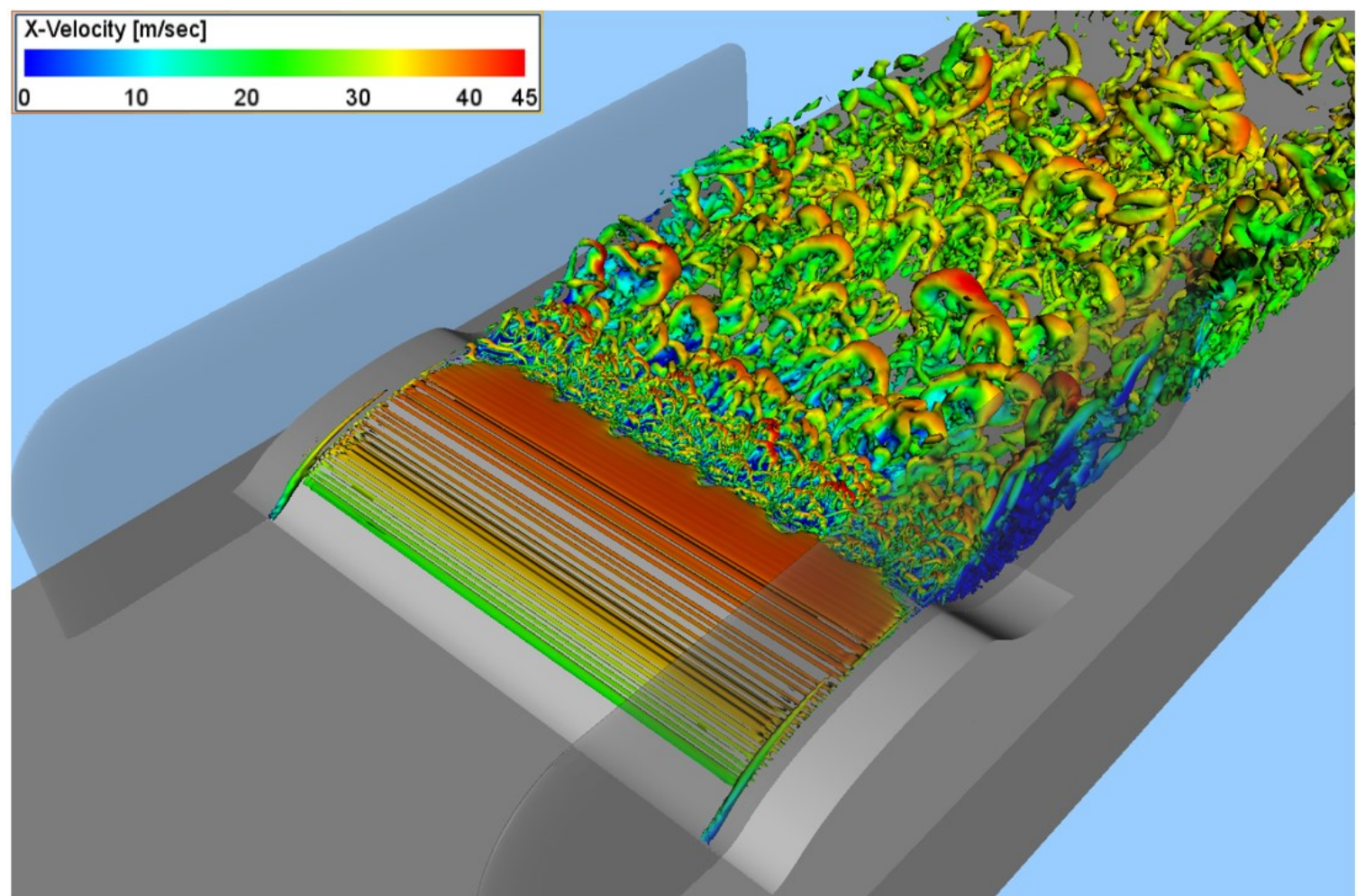

Figure 5. Instantaneous $\lambda_{2}$-isosurfaces for the steady excitation case $\left(C_{\mu}=0.24 \%\right)$.

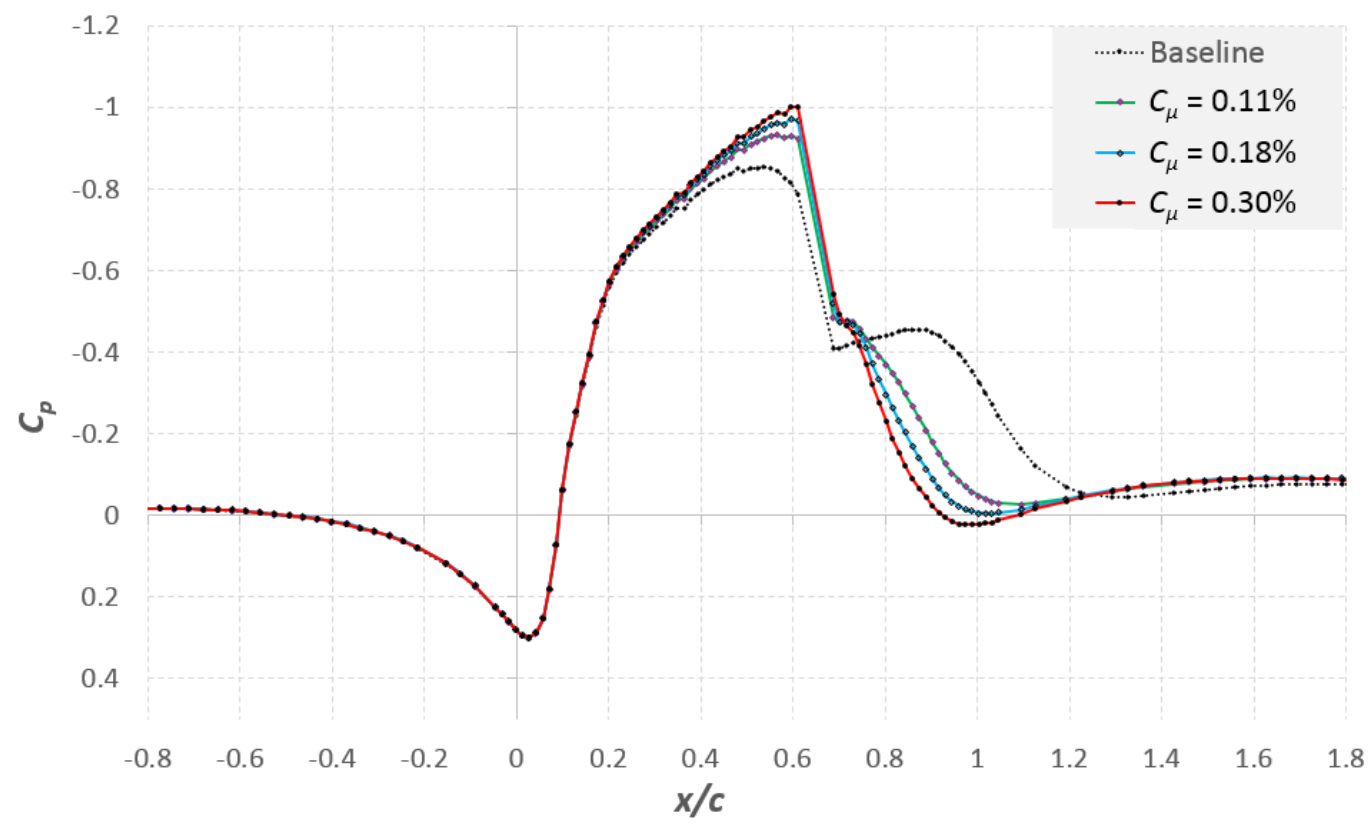

Figure 6. Variation of the $C_{p}$ distribution with the excitation amplitude (experiment). 


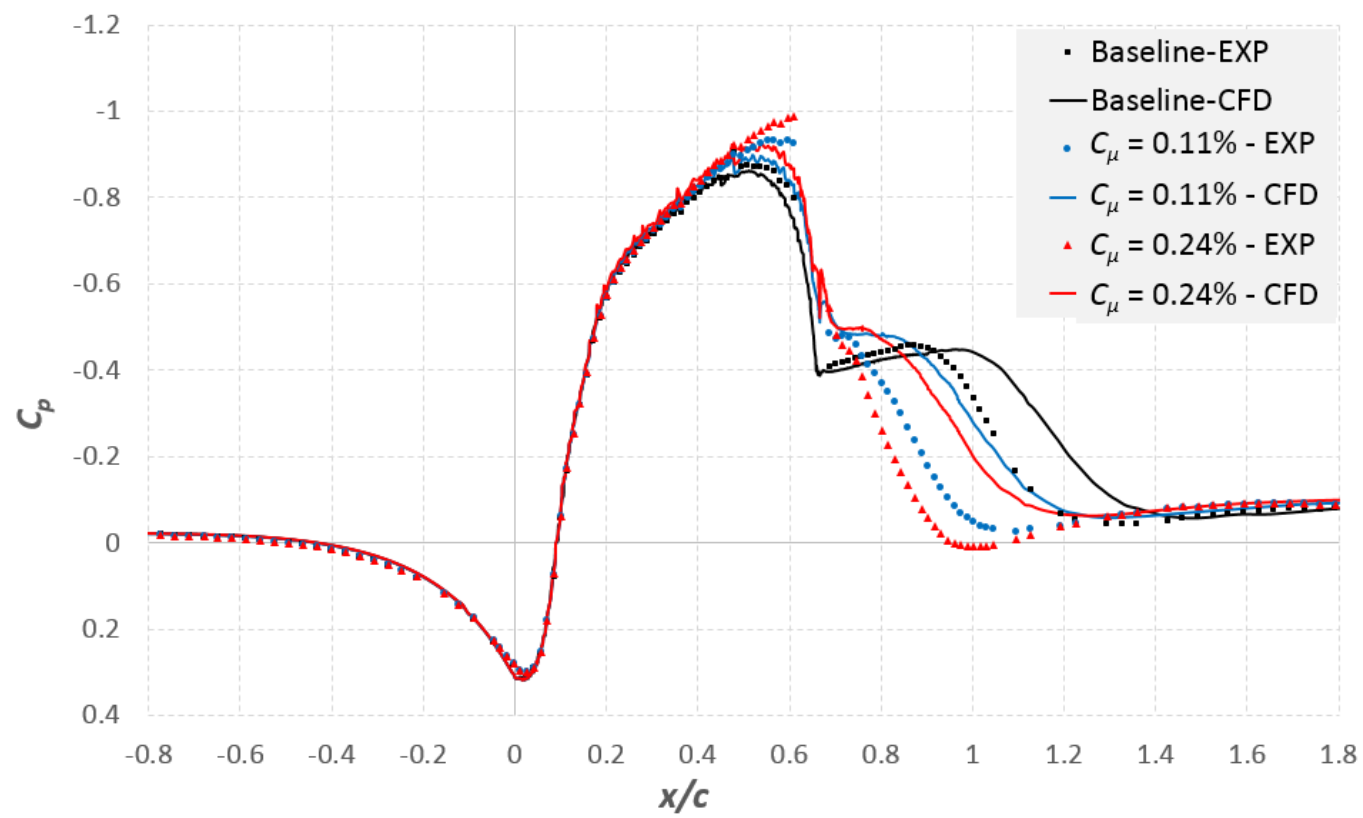

Figure 7. The effect of momentum coefficient with steady excitation.

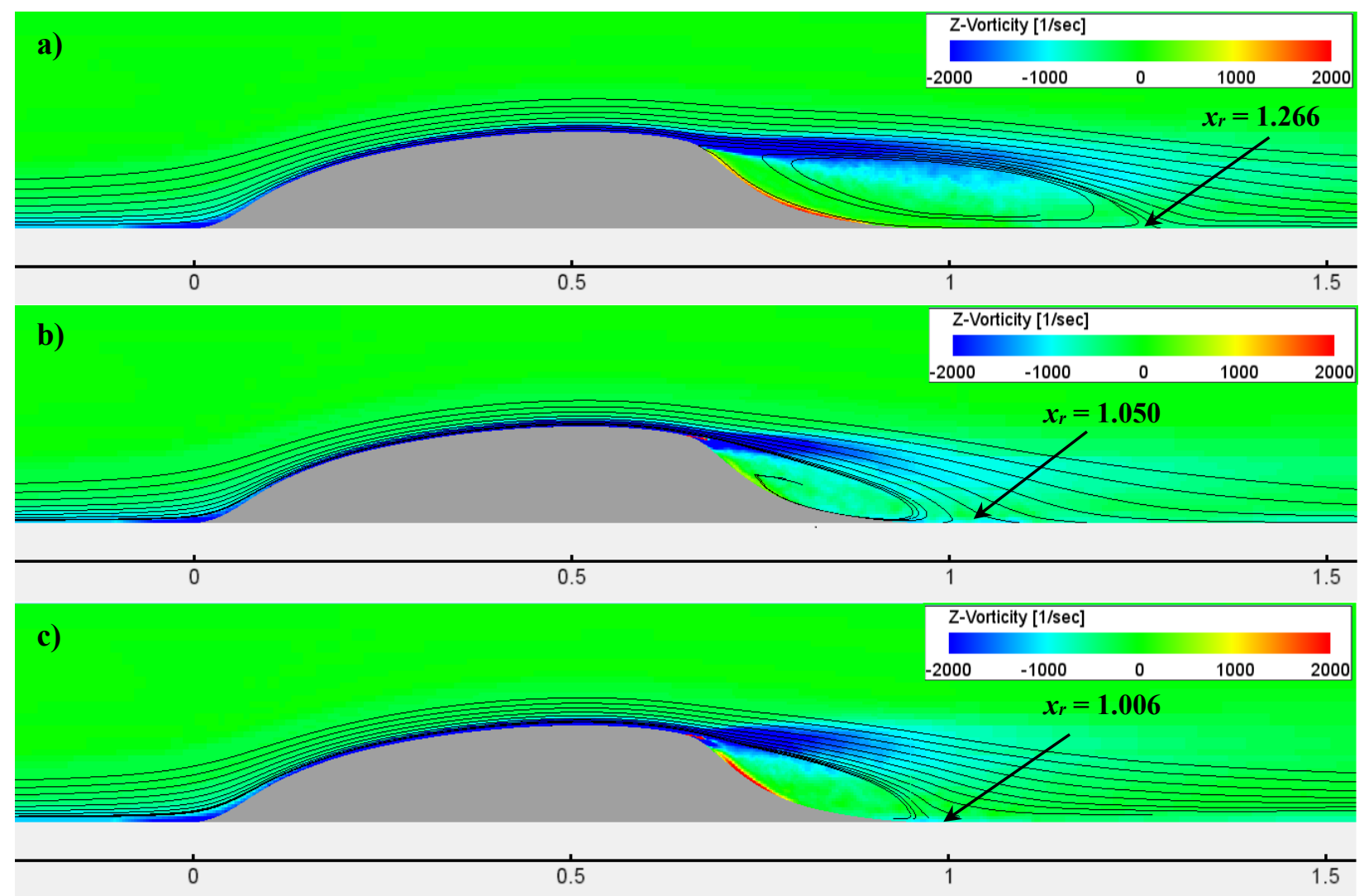

Figure 8. Spanwise vorticity contours: a) baseline, b) $C_{\mu}=0.11 \%$, c) $C_{\mu}=0.24 \%$, for steady excitation. 


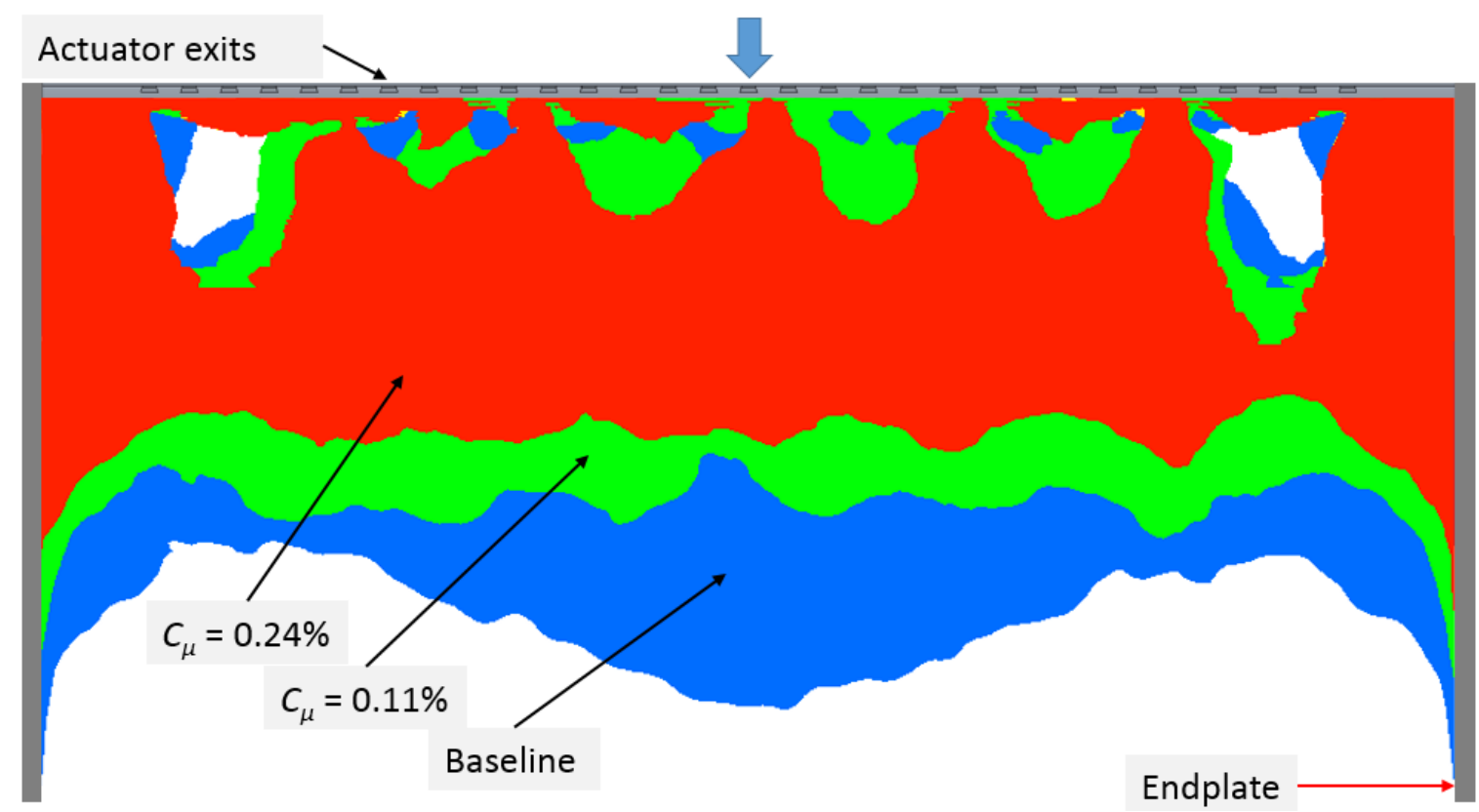

Figure 9. Variation of separation bubble with the steady excitation amplitude.

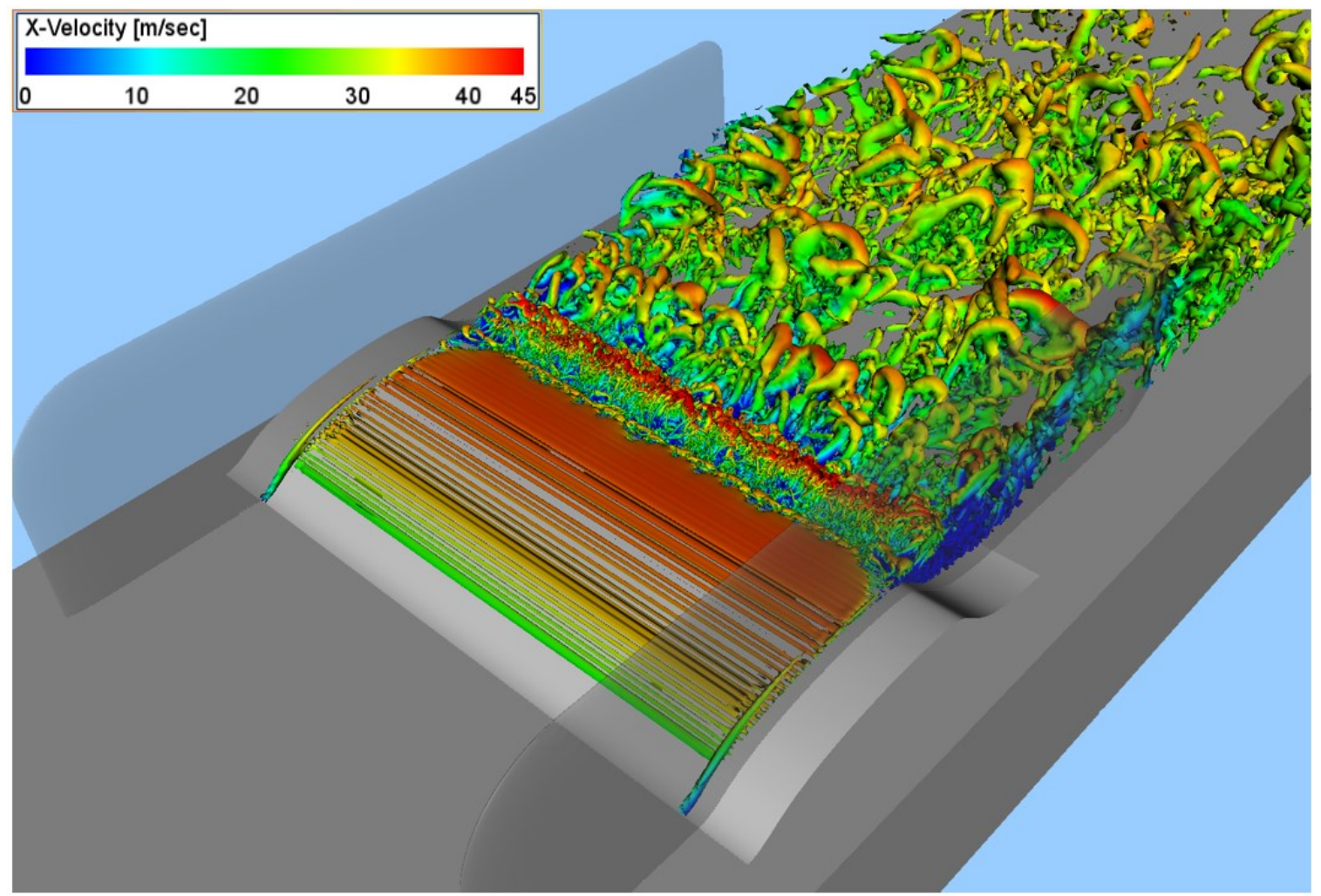

Figure 10. Instantaneous $\lambda_{2}$-isosurfaces for the unsteady excitation $\left(C_{\mu}=0.24 \%\right)$. 


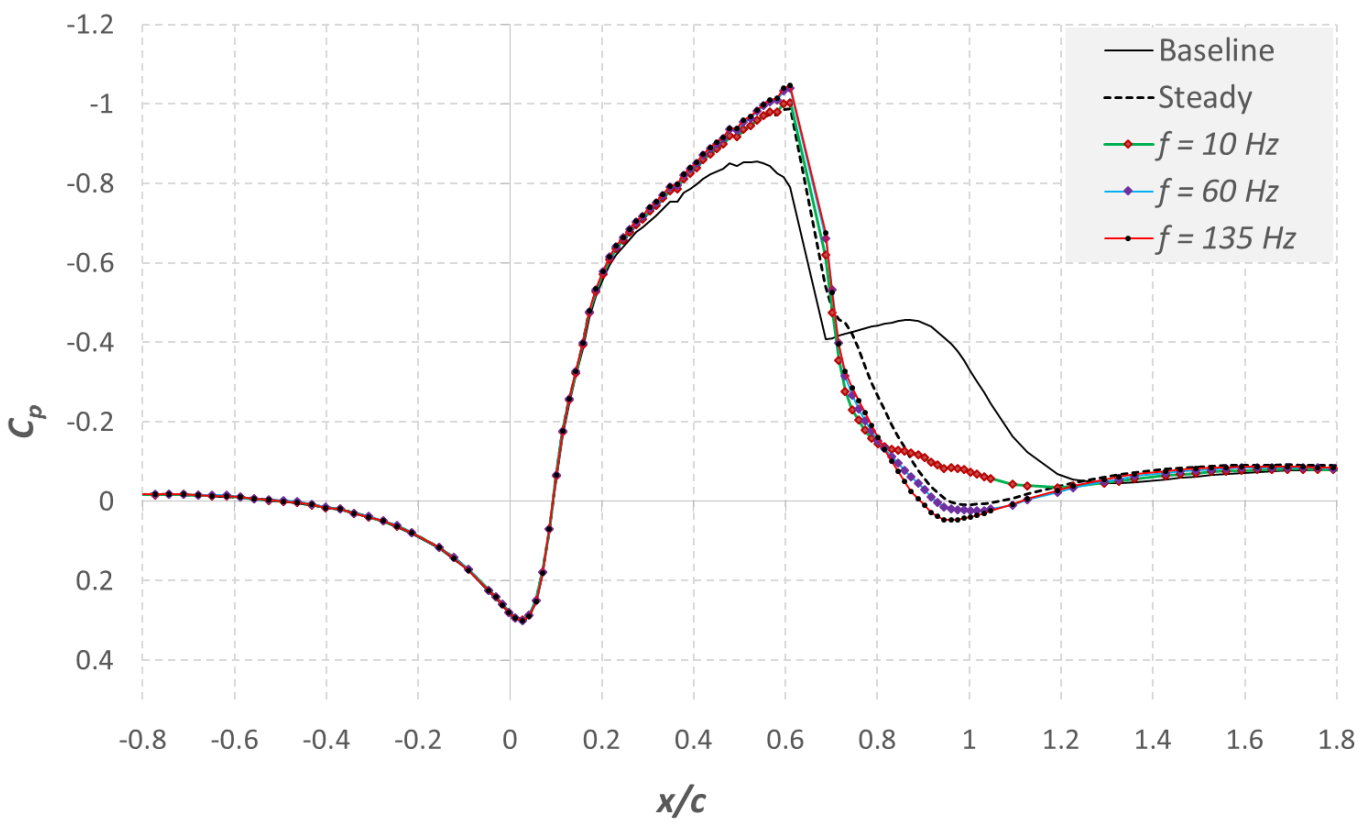

Figure 11. The effect of excitation frequency on the measured pressure distribution.

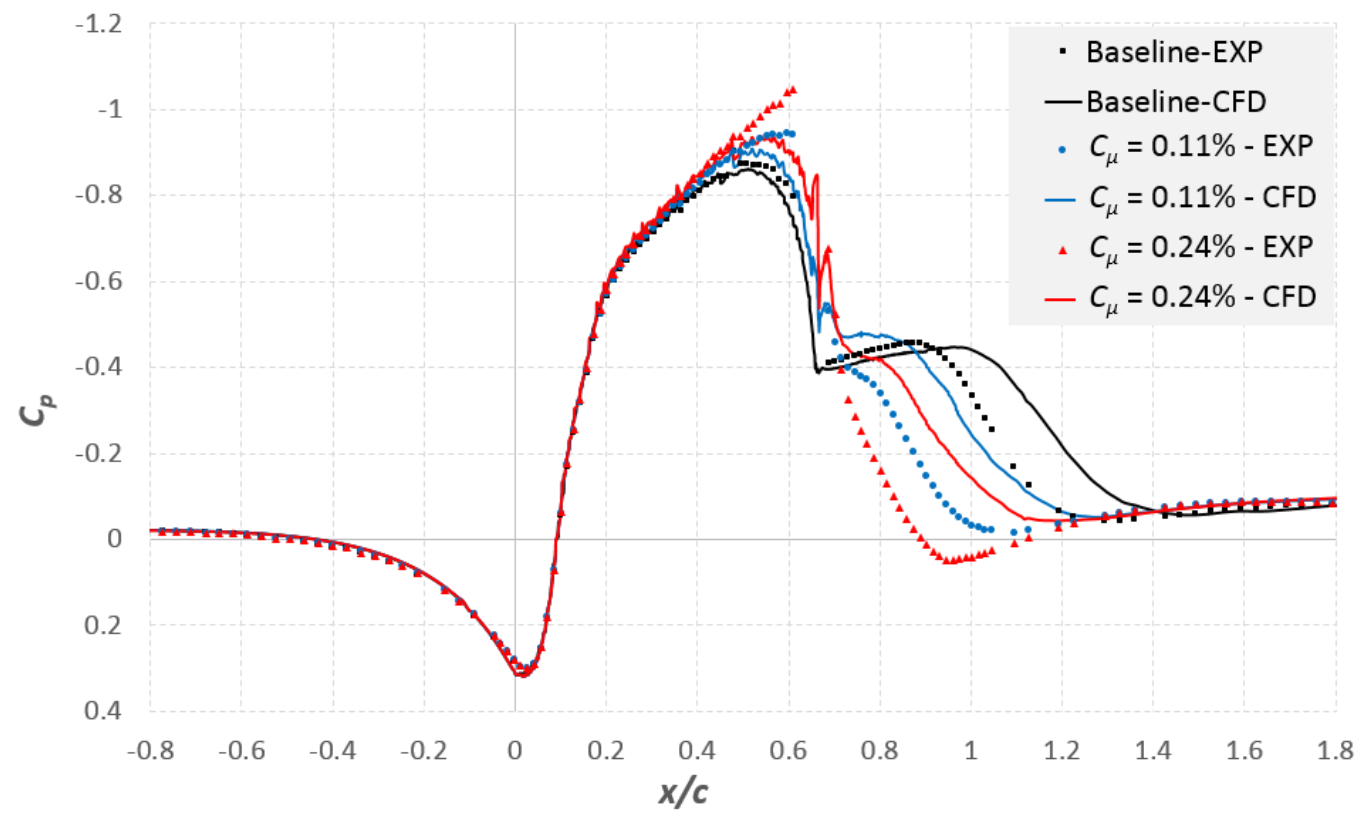

Figure 12. The effect of momentum coefficient for unsteady excitation. 


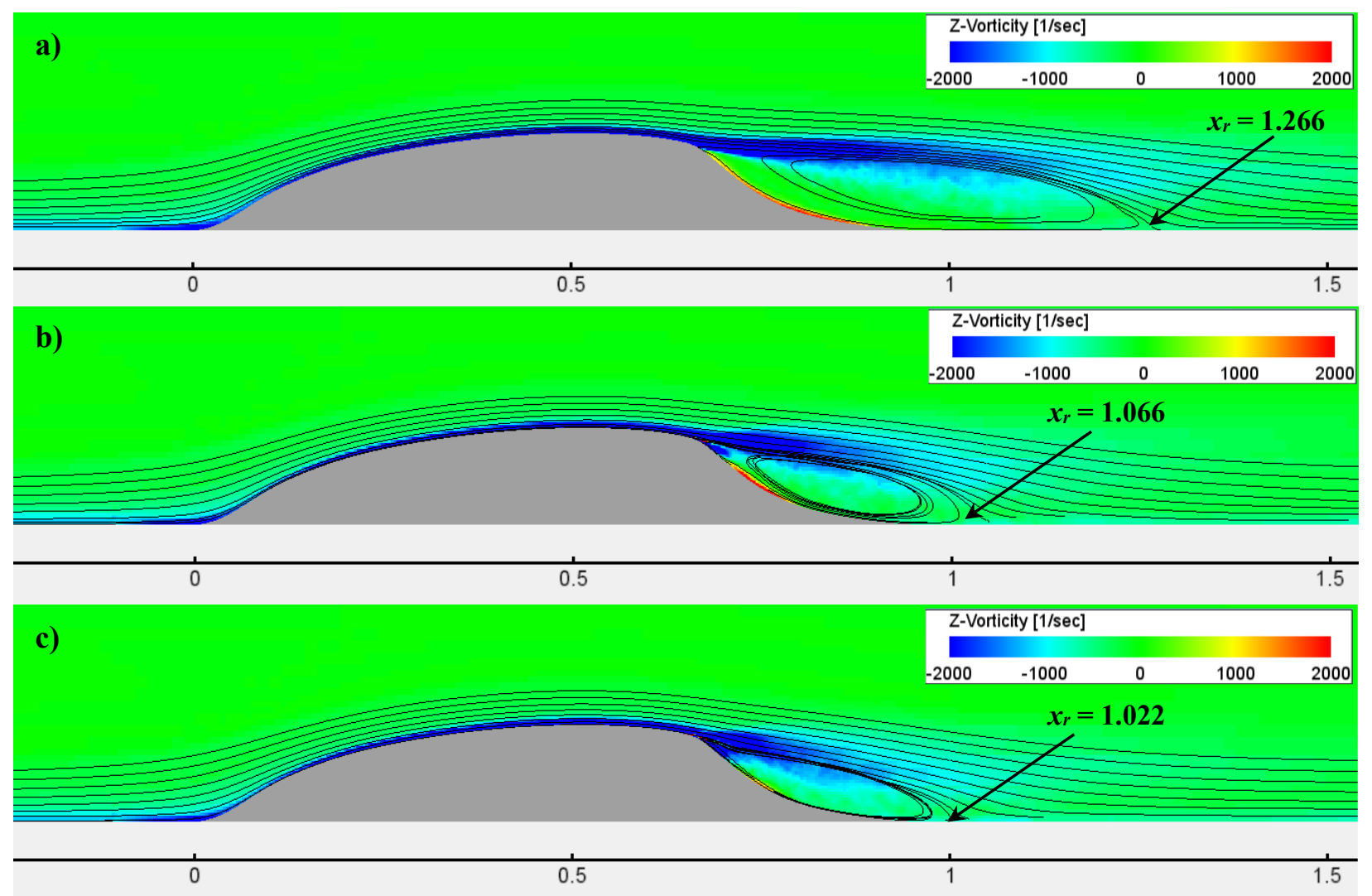

Figure 13. Spanwise vorticity contours: a) baseline, b) $C_{\mu}=0.11 \%$, c) $C_{\mu}=0.24 \%$ for unsteady excitation.

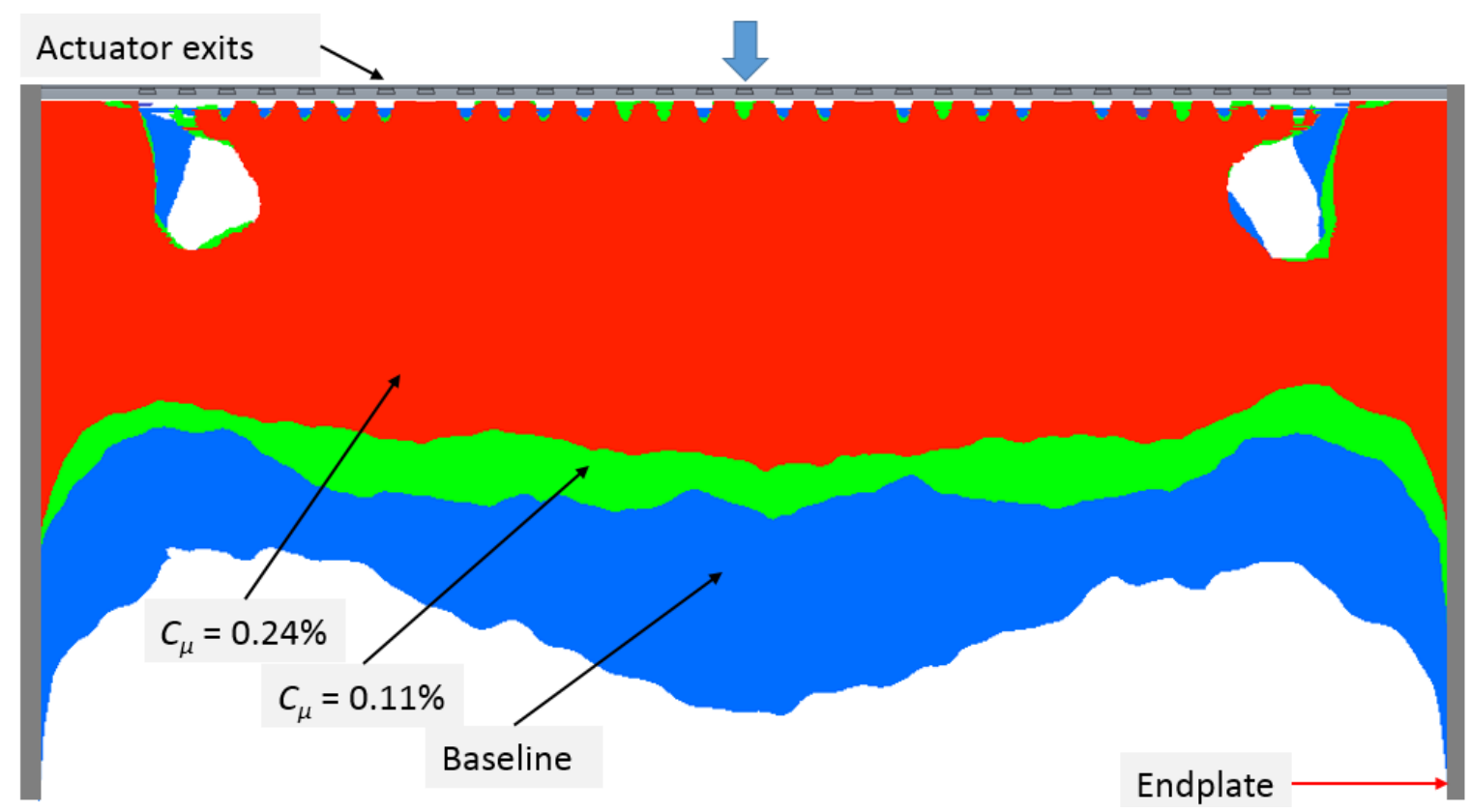

Figure 14. Variation of separation bubble with the unsteady excitation amplitude. 


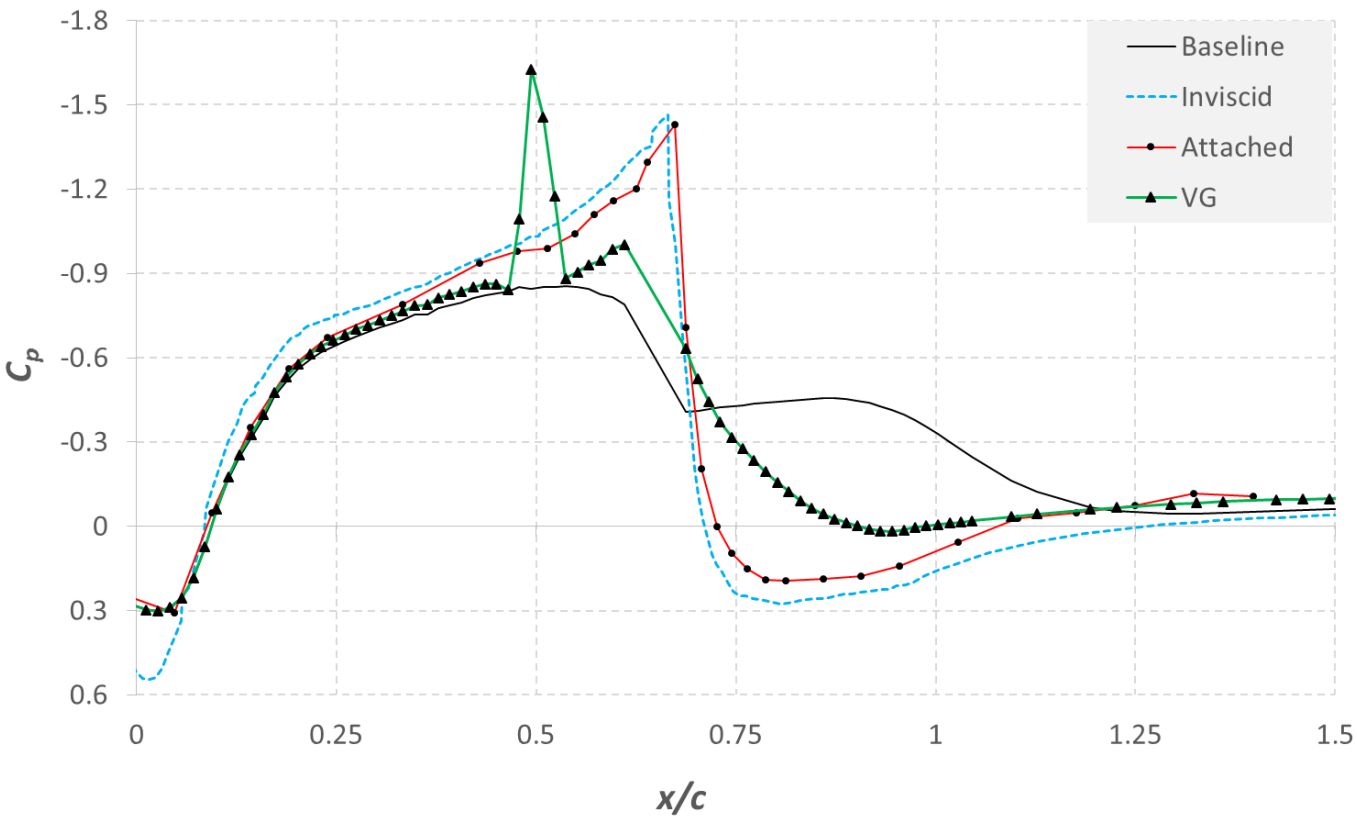

Figure 15. Centerline $C_{p}$ distributions for different flow control methods.

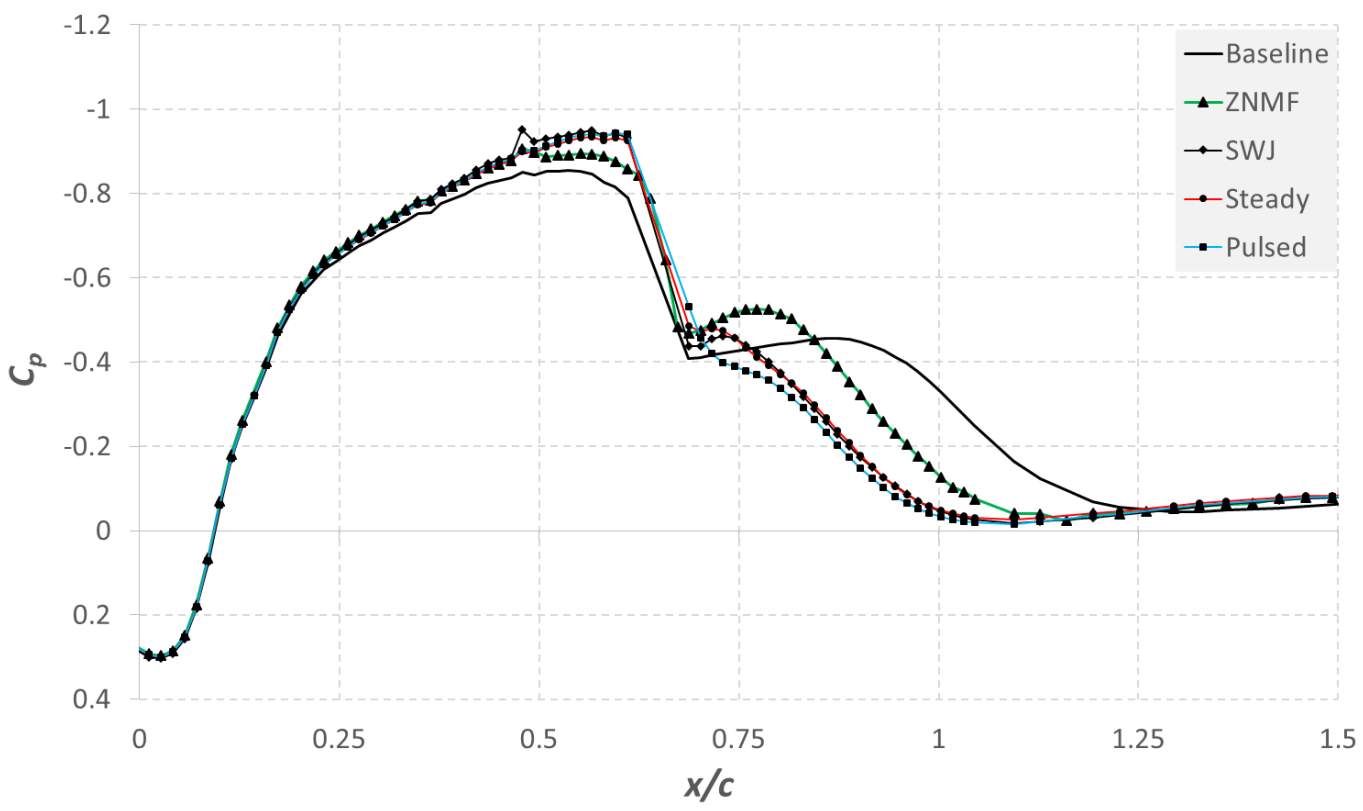

Figure 16. Comparison of different AFC methods at low excitation amplitude $\left(C_{\mu}=0.11 \%\right)$. 


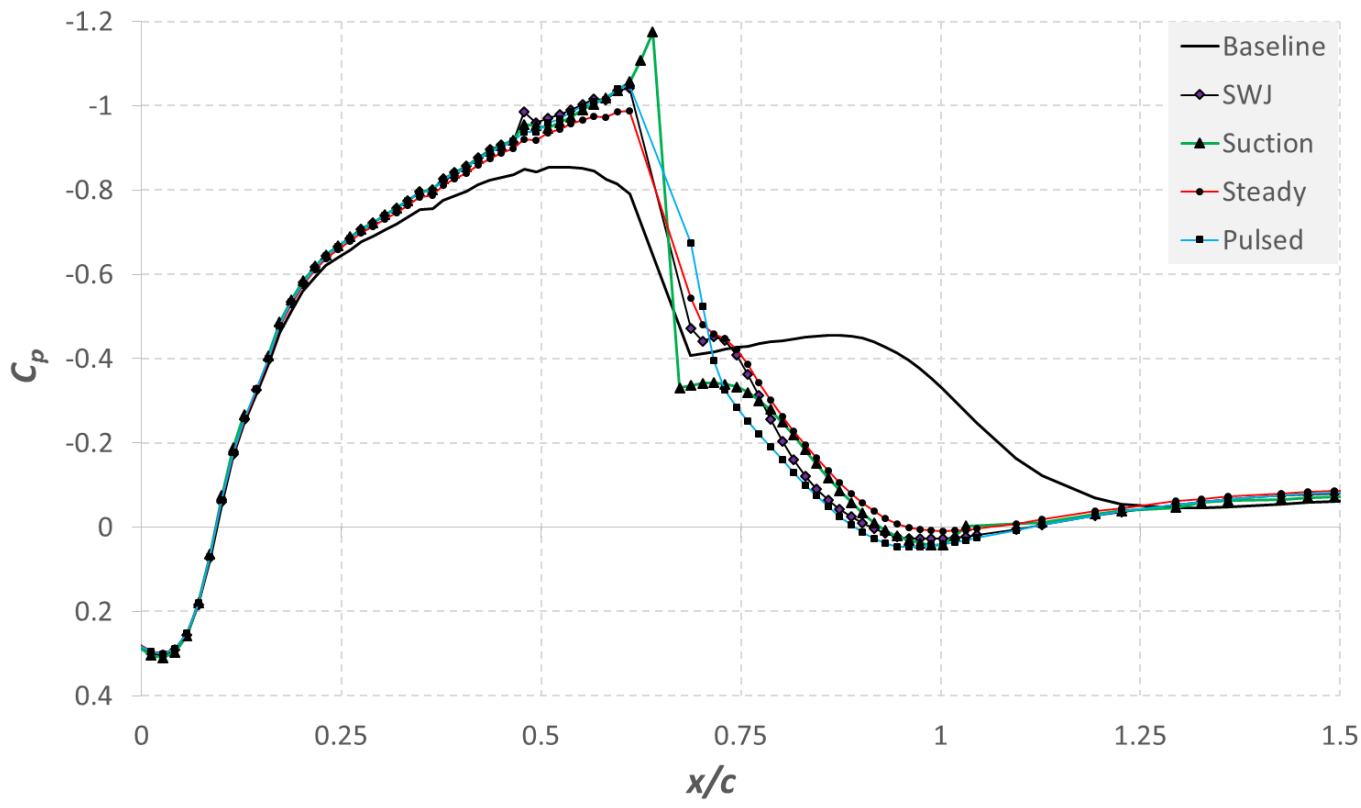

Figure 17. Comparison of different AFC methods at high excitation amplitude ( $\left.C_{\mu}=0.24 \%\right)$. 